\title{
A network pharmacology-based investigation on the bioactive ingredients and molecular mechanisms of Gelsemium elegans Benth against colorectal cancer
}

Wancai Que ${ }^{1 \dagger}$, Maohua Chen ${ }^{1 \dagger}$, Ling Yang ${ }^{2}$, Bingqing Zhang ${ }^{3}$, Zhichang Zhao ${ }^{1}$, Maobai Liu ${ }^{1}$, Yu Cheng ${ }^{1 *}$ and Hongqiang $\mathrm{Qiu}^{1,3^{*}}$ (D)

\begin{abstract}
Background: Colorectal cancer (CRC) remains one of the leading causes of cancer-related death worldwide. Gelsemium elegans Benth (GEB) is a traditional Chinese medicine commonly used for treatment for gastrointestinal cancer, including CRC. However, the underlying active ingredients and mechanism remain unknown. This study aims to explore the active components and the functional mechanisms of GEB in treating CRC by network pharmacology-based approaches.

Methods: Candidate compounds of GEB were collected from the Traditional Chinese Medicine@Taiwan, Traditional Chinese Medicines Integrated Database, Bioinformatics Analysis Tool for Molecular mechanism of Traditional Chinese Medicine, and published literature. Potentially active targets of compounds in GEB were retrieved from SwissTargetPrediction databases. Keywords "colorectal cancer", "rectal cancer" and "colon cancer" were used as keywords to search for related targets of CRC from the GeneCards database, then the overlapped targets of compounds and CRC were further intersected with CRC related genes from the TCGA database. The Cytoscape was applied to construct a graph of visualized compound-target and pathway networks. Protein-protein interaction networks were constructed by using STRING database. The DAVID tool was applied to carry out Gene Ontology and Kyoto Encyclopedia of Genes and Genome pathway enrichment analysis of final targets. Molecular docking was employed to validate the interaction between compounds and targets. AutoDockTools was used to construct docking grid box for each target. Docking and molecular dynamics simulation were performed by Autodock Vina and Gromacs software, respectively.

(Continued on next page)
\end{abstract}

\footnotetext{
* Correspondence: 86cy@163.com; hongqiangqiu@fjmu.edu.cn

${ }^{+}$Wancai Que and Maohua Chen contributed equally to this work.

'Department of Pharmacy, Fujian Medical University Union Hospital, 29 Xin

Quan Rd, Gulou, Fuzhou 350001, Fujian, People's Republic of China

Full list of author information is available at the end of the article
}

(c) The Author(s). 2021 Open Access This article is licensed under a Creative Commons Attribution 4.0 International License, which permits use, sharing, adaptation, distribution and reproduction in any medium or format, as long as you give appropriate credit to the original author(s) and the source, provide a link to the Creative Commons licence, and indicate if changes were made. The images or other third party material in this article are included in the article's Creative Commons licence, unless indicated otherwise in a credit line to the material. If material is not included in the article's Creative Commons licence and your intended use is not permitted by statutory regulation or exceeds the permitted use, you will need to obtain permission directly from the copyright holder. To view a copy of this licence, visit http://creativecommons.org/licenses/by/4.0/. The Creative Commons Public Domain Dedication waiver (http://creativecommons.org/publicdomain/zero/1.0/) applies to the data made available in this article, unless otherwise stated in a credit line to the data. 
(Continued from previous page)

Results: Fifty-three bioactive compounds were successfully identified, corresponding to 136 targets that were screened out for the treatment of CRC. Functional enrichment analysis suggested that GEB exerted its pharmacological effects against CRC via modulating multiple pathways, such as pathways in cancer, cell cycle, and colorectal cancer. Molecular docking analysis showed that the representative compounds had good affinity with the key targets. Molecular dynamics simulation indicated that the best hit molecules formed a stable protein-ligand complex.

Conclusion: This network pharmacology study revealed the multiple ingredients, targets, and pathways synergistically involved in the anti-CRC effect of GEB, which will enhance our understanding of the potential molecular mechanism of GEB in treatment for CRC and lay a foundation for further experimental research.

Keywords: Gelsemium elegans Benth, Colorectal cancer, Network pharmacology, Mechanism

\section{Background}

Colorectal cancer (CRC) continues to be one of the leading causes of mortality and morbidity worldwide despite the availability of reliable screening tools and effective therapies. It is the second most common cause of cancer death in the United States when men and women are combined [1]. According to the American Cancer Society's and GLOBOCAN estimates, it will be 147,950 and 1,931,590 new cases of CRC in the United States and the world for 2020, respectively [2,3]. The incidence and mortality of CRC are rapidly increasing particularly in developing countries, and it is estimated that the global burden of CRC increases by $60 \%$ over 2.2 million new cases and 1.1 million cancer death by 2030 [4]. Effective treatments used for CRC may include some combination of surgery, radiation therapy, chemotherapy, immunotherapy and targeted therapy [5-7]. However, the mortality is still relatively high because of delayed diagnosis, metastasis, and frequent recurrence. The 5year survival rate is less than $14 \%$ and unfortunately, more than $50 \%$ of CRC patients were diagnosed at an advanced stage [8]. Furthermore, using the most prevalent chemotherapy regimens has shown the limitations, a series of side effects commonly accompany patients, such as gastrointestinal reaction, bone marrow suppression, neurotoxicity, and abnormal liver or kidney function. It is of great significance to search for more effective alternative agents with low toxicity for patients.

Medicinal herbs are an important, yet often overlooked, a source for novel antineoplastic drugs. Indeed, many chemotherapeutics derived from plants, such as paclitaxel, vinblastine, and vincristine, have proven effective against different tumors. Sometimes as a complementary therapy, medicinal plants are widely used to treat several types of cancers, including CRC, with relatively fewer and milder side effects $[9,10]$. As an important source of alternative and complementary medicines, traditional Chinese medicine (TCM) has been widely reported to treat cancer [11-13]. In recent years, more and more herbs originating from TCM have attracted considerable attention as anti-CRC agents because of their therapeutic value and low toxicity $[9,14]$.

Gelsemium, as a genus of the Gelsemiaceae family, consists of three well-known species: Gelsemium elegans Benth. (GEB) (Fig. 1), native to Southeast Asia and China, and Gelsemium rankinii Small and Gelsemium sempervirens Ait, native to North America [15]. Although GEB is a toxic plant, it has long been used in Chinese folk medicine to treat many diseases, including pain, inflammation, and cancer [16, 17]. Alkaloids, isolated and purified from GEB, constitute the main active molecules of GEB and were profoundly studied for their biological activities in several pharmaceutical areas, including anti-inflammatory, antirheumatic, analgesic, immunomodulatory, and anti-tumor activities [16]. GEB has shown potential as a promising anti-tumor agent in clinical practice. Patients with severe primary liver cancer survived after treated with powder of GEB $(150 \mathrm{mg}$, Bid), resuting in tumor shrinkage and pain relief [18]. Notably, GEB has long been used as Chinese folk medicine in the treatment of CRC in Southern China [19]. In vitro study, cytotoxic effects on the tumor cells of

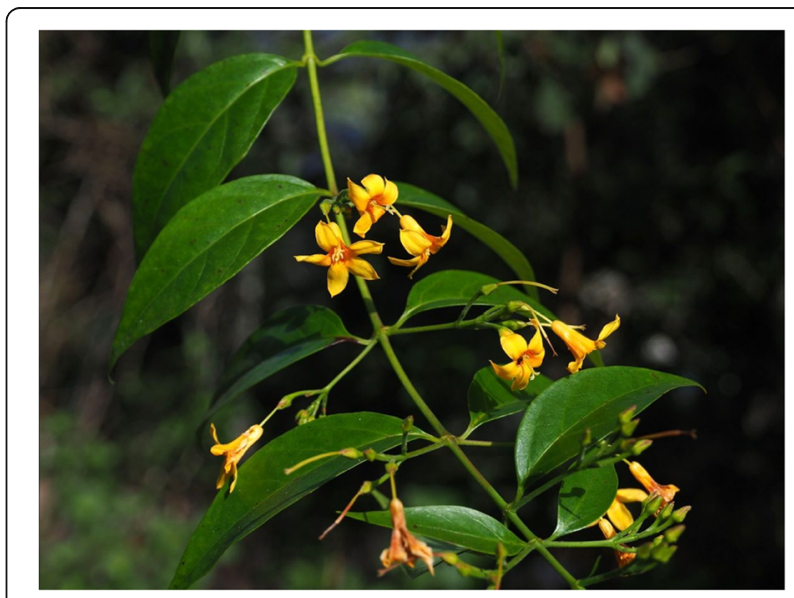

Fig. 1 Flowers and leaves from Gelsemium elegans Benth 
digestive system was observed in alkaloidal compounds from GEB [20]. Alkaloids of GEB could inhibit the proliferation and induced the apoptosis of the human colonic carcinoma cells [21, 22]. However, the potential active compounds and underlying molecular mechanisms of the anti-CRC effect of GEB remain unknown.

With the current merging of bioinformatics, network pharmacology-based analysis has become a robust method to systematically reveal the biological mechanisms of complex diseases and drug effects at the molecular level [23]. It integrates information science, systematic medicine, and is evolving as a promising strategy for the next-generation mode of drug discovery and development for traditional medicine. Compared with traditionally experimental pharmacology research, network pharmacology focuses on analyzing multiple target regulation of multiple chemical components, so it is particularly suitable for the interpretation of the mechanism of TCM [24]. Hence, the present study aimed to reveal the potentially active ingredients against $\mathrm{CRC}$ and predict the underlying action mechanism of GEB by employing a network pharmacological method.

\section{Methods}

\section{Potentially active compounds in GEB}

The information about compounds of GEB was obtained from the published literature $[16,25]$ and the following online databases: (1) Traditional Chinese Medicines Integrated Database (TCMID) (http://11 9.3.41.228:8000/tcmid/), which is a comprehensive database to provide information and bridge the gap between TCM and modern life sciences [26]. (2) Bioinformatics Analysis Tool for Molecular mechanism of Traditional Chinese Medicine (BATMAN-TCM) (http://bionet.ncpsb.org/batman-tcm/), which is the first online bioinformatics analysis tool specially designed for the research of molecular mechanism of TCM [27]. (3) Traditional Chinese Medicine Database@ Taiwan (http://tcm.cmu.edu.tw/zh-tw/), the world's largest TCM database for drug screening in silico [28]. Then all compounds of the herbal medicine were determined by removing the duplicate compounds.

The candidate active compounds were further filtered by meeting at least two of five features of drug-likeness (Lipinski, Ghose, Veber, Egan, and Muegge) and combining bioavailability score $\geq 30 \%$ as suggested by the http://www.swissadme.chwebsite, which allows to compute physicochemical descriptors as well as to predict ADME parameters, pharmacokinetic properties, druglike nature and medicinal chemistry friendliness of one or multiple small molecules to support drug discovery [29].

\section{Targets prediction of compounds in GEB}

As a popular online server, SwissTargetPrediction (http://www.swisstargetprediction.ch) provides information on chemical substances, biological activities, and allows to estimate the most probable macromolecular targets of a small molecule [30]. 3D molecular structure files of each ingredient that could be retrieved from PubChem (https://pubchem.ncbi.nlm.nih.gov/) were imported into SwissTargetPrediction for identification of potential drug target in humans. The targets of ingredients acquired from SwissTargetPrediction with probability $\geq 0.1$ were chosen as potential targets in this study after removing the repeated targets. Those compounds without target information were excluded.

\section{Targets of CRC}

Information on CRC-associated target genes was collected from the following resources. The different genes involved in CRC were gathered from GeneCards (https://www.genecards.org/), which is a searchable, integrative database that provides comprehensive, userfriendly information on all annotated and predicted genes involved in human diseases [31]. Keywords "colorectal cancer", "rectal cancer", and "colon cancer", were used to search through the database, which identified 33, 505 genes with a disease relevance score $\geq 10$. Then, the putative target genes of GEB were mapped to the CRCassociated target genes. The candidate anti-CRC targets of GEB were visualized by overlapping the above targets with a Venn diagram.

\section{Validation of candidate targets in the cancer genome atlas (TCGA) database}

CRC related genes from The Cancer Genome Atlas (TCGA) database of ONCOMINE (https://www. oncomine.org/) were used to validate candidate targets of GEB against CRC. Firstly, the top 10\% overexpression and under-expression mRNA genes form CRC samples (Cecum Adenocarcinoma vs. Normal, Colon Adenocarcinoma vs. Normal, Colon Mucinous Adenocarcinoma vs. Normal, Rectal Adenocarcinoma vs. Normal, Rectal Mucinous Adenocarcinoma vs. Normal, and Rectosigmoid Adenocarcinoma vs. Normal) were downloaded from the TCGA database of ONCOMINE. Subsequently, a Venn diagram was used to intersect the candidate targets of GEB against CRC genes obtained from the TCGA database.

\section{Protein-protein interaction (PPI) data}

Core regulatory genes can be identified by exploring the protein-protein interaction (PPI). PPI information can be obtained from the STRING database (https://stringdb.org/), which covers abundant information regarding known and predicted protein-protein interactions of 
different species [32]. In this study, high confidence score $>0.7$ were reserved and the species was only limited to "Homo sapiens", then the validated targets were submitted to STRING. Finally, PPI data were extracted. The top 20 proteins with a higher level of degrees were considered as the center targets for GEB in the treatment for CRC.

\section{Cluster analysis}

There are some closely connected regions of molecular complexes in large PPI networks, which are named topology modules or clusters. Cluster analysis is a classification method that involves interconnected regions showing the inherent laws in the network. In this study, significant cluster modules from the constructed PPI network were selected using the Molecular Complex Detection (MCODE), a plug-in of Cytoscape, which was used to detect densely connected regions and cluster analysis in the PPI network [33]. The criteria settings were set as follows: node score cutoff $=0.2$; $\mathrm{K}$-core $=2$; and degree of cutoff $=2[34]$.

\section{GO and KEGG pathway enrichment analysis}

To explore the gene functions, the Database for Annotation, Visualization and Integrated Discovery (DAVID, https://david.ncifcrf.gov/.ver.6.8) which provides a systematic and comprehensive set of functional annotation tools for investigators to understand the biological meanings behind a large list of genes [35], was applied to perform Gene Ontology (GO) and Kyoto encyclopedia of genes and genome (KEGG) pathway enrichment of proteins in the PPI network analyses.The species was only limited to "Homo sapiens". Those GO and KEGG pathway terms with only False Discovery Rate (FDR) $<0.01$ were considered to be significantly enriched. As for enrichment analysis, the results of enriched GO terms of biological process (BP), cellular component (CC), and molecular function (MF) were visualized by the $\mathrm{R}$ software package (3.5.2), as well as the bubble chart of KEGG pathway enrichment.

\section{Network construction}

Four networks were constructed as follows: (1) Compounds-compound targets network of GEB was constructed by connecting chemical compounds with corresponding targets; (2) Potential compounds-targets network of GEB against CRC; (3) Potential compoundstargets-pathways network of GEB against CRC; (4) PPI network of the potential targets of GEB against CRC. The PPI network was completed directly on STRING. The other 3 networks were constructed using the network visualization software Cytoscape (http://cytoscape. org/.ver.3.7.2), which is an open-source software platform suitable for visualizing intermolecular interactions networks and biological pathways [36]. Furthermore, Cytoscape can be used to integrate and analyze these networks with annotations, gene expression profiles, and other complicated data. Three parameters can be calculated to evaluate the topological coefficients of each node. "Degree" represents the number of edges connected to a node; "Betweenness" is defined as the number of times a node act as a bridge along the shortest paths between pairs of other nodes; "Closeness" is the inverse of the sum of the shortest paths from a node to other nodes in the network.

\section{Active compounds-targets docking}

Ten compounds were selected from the core compounds of GEB and docked with six proteins selected from the center targets to verify the accuracy of the main compounds and their corresponding predicted targets. The candidate compound and the crystal structure of the target protein were downloaded from the PubChem database and RCSB protein data (http://www.rcsb.org), respectively. The latter preferably selects a model with ligand binding smaller than $3 \AA$, and then dehydration, hydrogenation, and separation of ligands were carried out by importing the crystal structure into the Pymol 2.4.1 Software (https://pymol.org/2/); then AutoDockTools 1.5.6 was used to construct the docking grid box of crystal structure for each target [37]. Docking was done by Autodock Vina 1.1.2 software, and the molecules with the lowest binding energy in the docking conformation were selected to observe the binding effect by comparing with the original ligands and intermolecular interactions (such as hydrophobicity, cation- $\pi$, anion- $\pi$, $\pi-\pi$ stacking, hydrogen bonding, etc.). The proteins with the original ligands were specified docking at a domain of the protein, and amino acid residues in the domain were targeted for evaluating the interaction. The number of grid points in the three dimensions (NPTS) used in this study were 4040400.375 . Since RCSB did not find the effective crystal structure of the CCND1 binding ligand, direct docking was performed with grid center 24.68313 .20561 .426 .

\section{Molecular dynamics simulation}

In order to analyze the binding affinities of the best hit molecules (gelsesyringalidine and CDK2) after docking, a $10 \mathrm{~ns}$ atomistic molecular dynamics (MD) simulation of selected protein-ligand complex was conducted. In the present study the NVIDIA RTX 1060 GPU accelerated GROMACS 2021 software, running over Linux ubuntu 20.04 operating system supported by AMD R5 3600 processor was used. The Charmm36 force field was used to generate protein topology. The ligand topology and parameters required for MD simulation were generated by using CGenFF server. The TIP3P water model was used 
for solvating each systems followed by neutralization with appropriate numbers of $\mathrm{Na}^{+}$and $\mathrm{Cl}^{-}$. Then energy of each system was minimized by using the steepest descent minimization algorithm with maximum 50,000 steps and $<10.0 \mathrm{~kJ} / \mathrm{mol}$ force. Position restrains have been applied to receptor and ligand of the each systems for $100 \mathrm{ps}$ throughout heating (300 K) utilizing NVT (No. of atoms, Volume, Temperature) ensemble with leap-frog integrator, a time step of $2 \mathrm{fs}$ and LINCS holonomic constraints.NPT (No. of atoms, Pressure, Temperature) ensemble has been applied at temperature $(300 \mathrm{~K})$ for $100 \mathrm{ps}$ using a time step of $2 \mathrm{fs}$ for NPT equilibration phase. After the energy minimization and equilibration of all systems, MD production run has been executed without any restrain for $10 \mathrm{~ns}$ with a time step of $2 \mathrm{fs}$, and after every $10 \mathrm{ps}$ coordinates of the structure have been saved. After the completion of $10 \mathrm{~ns}$ MD simulation, the trajectories have been used for various dynamics analysis such as root mean square deviation (RMSD) and root mean square fluctuation (RMSF). These were compared with the primitive ligand complex.

\section{Statistics}

Benjamini-Hochberg correction was performed for multiple testing, and adjusted value $<0.05$ was set as the threshold. False Discovery Rate (FDR) $<0.01$ was deemed as significant enriched in GO and KEGG analysis.

\section{Results}

\section{Potentially active compounds and targets in GEB}

Using the BATMAN-TCM and TCM@Taiwan databases, we collected a total of 97 compounds in GEB. Eventually, based on the filtering rules, $(\mathrm{OB} \geq 30 \%$ and the features of drug-likeness), 56 potentially active compounds were identified from a total compound in GEB. Details of the 56 potentially bioactive compounds are provided in Table 1. By using SwissTargetPrediction for target prediction, 729 potential targets were found for GEB (Table S1). The compounds-compound targets network as shown in Fig. 2, included 785 nodes: 56 active compound nodes and 729 target nodes, and 3747 edges. The top 5 compound nodes with the greatest number of edges included Humantenine, n-desmethoxtrankinidine, 19-(Z)-Akuammidine, 19 - Z- akuammidine, and NDesmethoxyhumantenine. Three topological features of these compounds exhibited mean values of degree, node betweenness, and closeness were 111.2, 0.04769 and 0.3723 , respectively. The top 5 target nodes with highest degree were LRRK2, JAK1, DRD3, EGFR, and IGF1R. nidine, 19-(Z)-Akuammidine, 19 - Z- akuammidine, and $\mathrm{N}$ - Desmethoxyhumantenine. Three topological features of these targets exhibited mean values of degree, node betweenness, and closeness were 29, 0.01554 and 0.4025 , respectively.

Red circle nodes stand for the target genes. Green nodes stand for compounds in GEB.

The size of the node represents the number of the degrees.

\section{Targets of compounds in GEB against CRC}

CRC-related target genes were retrieved from GeneCards, 33,505 potential target genes related to CRC were obtained. A total of 1893 potential target genes were included as candidate genes with a disease relevance score $\geq 10$. The details about the selected 1893 candidate target genes are described in Table S2. Then, the predictive target genes of GEB were overlapped with the candidate target genes. By intersecting the 729 target genes of GEB with the 1893 candidate target genes related to CRC, we obtained 272 intersections of target genes excluding any duplicate targets (Table S3, Fig. 3A). These intersections were considered potential candidate targets of GEB against CRC.

The overlapping number of GEB target genes and CRC (A), and the number of overlapping GEB candidate genes against $\mathrm{CRC}$ and $\mathrm{CRC}$ genes from The Cancer Genome Atlas (TCGA) database (B).

\section{Candidate therapeutic targets validation in the TCGA database}

A total of 8926 CRC related genes were identified in 215 tumor samples and 22 adjacent non-tumor samples from the TCGA database. These genes were significantly over-expressed and under-expressed genes (Table S4). Comparison of the 272 potential GEB candidate target genes against $\mathrm{CRC}$ with the $8926 \mathrm{CRC}$ related genes from TCGA revealed 136 common genes (Fig. 3B). These 136 common genes were considered key potential anti-CRC target genes of GEB (Table S5). As shown in Fig. 4, the compounds and targets related to CRC network analysis indicated that the top 5 compound nodes with the greatest number of edges included gelsesyringalidine, hydroxygenkwanin, Gelegamine E, oxoglaucine, and $19 \alpha$-hydroxygelsamydine. Three topological features of these compounds exhibited mean values of degree, node betweenness, and closeness were 34.2, 0.08659 and 0.4206 , respectively. The top 5 gene nodes with the greatest number of edges included EGFR, IGF1R, ABCB1, DPP4, and PARP1. Three topological features of these compounds exhibited mean values of degree, node betweenness, and closeness were 25.4, 0.03637 and 0.4359 , respectively.

Red circle nodes stand for the potential target genes related to CRC. Green nodes stand for potential active compounds in GEB for treating CRC. The size of the node represents the number of the degrees. 
Table 1 Information of the active compounds in GEB for network analysis

\begin{tabular}{|c|c|c|c|c|c|}
\hline NO & Name & $\begin{array}{l}\text { Compound } \\
\text { CID }\end{array}$ & MW & MF & Source \\
\hline 1 & N- Desmethoxyrankinidine & $5,316,594$ & 310.4 & $\mathrm{C}_{19} \mathrm{H}_{22} \mathrm{~N}_{2} \mathrm{O}_{2}$ & TCMID, References $[16,24]$ \\
\hline 2 & 11- Methoxygelsemamide & $5,319,437$ & 355.4 & $\mathrm{C}_{21} \mathrm{H}_{25} \mathrm{NO}_{4}$ & TCM-Taiwan, References $[16,24]$ \\
\hline 3 & Gelsevirine & $14,217,344$ & 352.4 & $\mathrm{C}_{21} \mathrm{H}_{24} \mathrm{~N}_{2} \mathrm{O}_{3}$ & $\begin{array}{l}\text { TCMID, BATMAN-TCM, TCM-Taiwan, Refer- } \\
\text { ences }[16,24]\end{array}$ \\
\hline 4 & Gelsenicine & $21,123,652$ & 326.4 & $\mathrm{C}_{19} \mathrm{H}_{22} \mathrm{~N}_{2} \mathrm{O}_{3}$ & References $[16,24]$ \\
\hline 5 & Gelsedine & $21,589,070$ & 328.4 & $\mathrm{C}_{19} \mathrm{H}_{24} \mathrm{~N}_{2} \mathrm{O}_{3}$ & $\begin{array}{l}\text { TCMID, BATMAN-TCM, TCM-Taiwan, Refer- } \\
\text { ences }[16,24]\end{array}$ \\
\hline 6 & 19 - Z- akuammidine & $44,583,830$ & 352.4 & $\mathrm{C}_{21} \mathrm{H}_{24} \mathrm{~N}_{2} \mathrm{O}_{3}$ & References $[16,24]$ \\
\hline 7 & Dihydrokoumine & $5,316,727$ & 308.4 & $\mathrm{C}_{20} \mathrm{H}_{24} \mathrm{~N}_{2} \mathrm{O}$ & BATMAN-TCM, References $[16,24]$ \\
\hline 8 & 19- (R)- Hydroxydihydrokoumine & $50,278,496$ & 324.4 & $\mathrm{C}_{20} \mathrm{H}_{24} \mathrm{~N}_{2} \mathrm{O}_{2}$ & $\begin{array}{l}\text { TCMID, BATMAN-TCM, TCM-Taiwan, Refer- } \\
\text { ences }[16,24]\end{array}$ \\
\hline 9 & 19- (S)- Hydroxydihydrokoumine & $5,318,193$ & 324.4 & $\mathrm{C}_{20} \mathrm{H}_{24} \mathrm{~N}_{2} \mathrm{O}_{2}$ & References $[16,24]$ \\
\hline 10 & $\mathrm{~N}$ - Desmethoxyhumantenine & $5,316,593$ & 324.4 & $\mathrm{C}_{20} \mathrm{H}_{24} \mathrm{~N}_{2} \mathrm{O}_{2}$ & TCMID, BATMAN-TCM, Reference [24] \\
\hline 11 & 15- Hydroxyhumantenine & $101,606,434$ & 370.4 & $\mathrm{C}_{21} \mathrm{H}_{26} \mathrm{~N}_{2} \mathrm{O}_{4}$ & TCMID, References $[16,24]$ \\
\hline 12 & Humantenmine & 158,212 & 326.4 & $\mathrm{C}_{19} \mathrm{H}_{22} \mathrm{~N}_{2} \mathrm{O}_{3}$ & $\begin{array}{l}\text { TCMID, BATMAN-TCM, TCM-Taiwan, Reference } \\
\text { [24] }\end{array}$ \\
\hline 13 & 11-Hydroxyrankinidine & $5,318,332$ & 356.4 & $\mathrm{C}_{20} \mathrm{H}_{24} \mathrm{~N}_{2} \mathrm{O}_{4}$ & TCMID, References $[16,24]$ \\
\hline 14 & 11-Hydroxyhumantenine & $5,318,224$ & 370.4 & $\mathrm{C}_{21} \mathrm{H}_{26} \mathrm{~N}_{2} \mathrm{O}_{4}$ & TCMID, References $[16,24]$ \\
\hline 15 & 11-Methoxyhumantenine & $44,583,832$ & 384.5 & $\mathrm{C}_{22} \mathrm{H}_{28} \mathrm{~N}_{2} \mathrm{O}_{4}$ & TCMID, BATMAN-TCM, References $[16,24]$ \\
\hline 16 & 19- (S)- Hydroxydihydrogelsevirine & $5,318,192$ & 370.4 & $\mathrm{C}_{21} \mathrm{H}_{26} \mathrm{~N}_{2} \mathrm{O}_{4}$ & References $[16,24]$ \\
\hline 17 & Gelseoxazolidinine & $102,297,300$ & 428.5 & $\mathrm{C}_{23} \mathrm{H}_{28} \mathrm{~N}_{2} \mathrm{O}_{6}$ & References $[16,24]$ \\
\hline 18 & Gelselegine & $10,948,335$ & 358.4 & $\mathrm{C}_{20} \mathrm{H}_{26} \mathrm{~N}_{2} \mathrm{O}_{4}$ & TCMID, References $[16,24]$ \\
\hline 19 & 19a-Hydroxygelsamydine & $102,003,053$ & 524.6 & $\mathrm{C}_{29} \mathrm{H}_{36} \mathrm{~N}_{2} \mathrm{O}_{7}$ & References $[16,24]$ \\
\hline 20 & Gelsamydine & $5,317,540$ & 508.6 & $\mathrm{C}_{29} \mathrm{H}_{36} \mathrm{~N}_{2} \mathrm{O}_{6}$ & TCMID, References $[16,24]$ \\
\hline 21 & Gelegamine $\mathrm{E}$ & $101,467,881$ & 370.4 & $\mathrm{C}_{20} \mathrm{H}_{22} \mathrm{~N}_{2} \mathrm{O}_{5}$ & References $[16,24]$ \\
\hline 22 & Gelegamine A & $101,467,877$ & 384.4 & $\mathrm{C}_{21} \mathrm{H}_{24} \mathrm{~N}_{2} \mathrm{O}_{5}$ & References $[16,24]$ \\
\hline 23 & Gelegamine B & $101,467,878$ & 384.4 & $\mathrm{C}_{21} \mathrm{H}_{24} \mathrm{~N}_{2} \mathrm{O}_{5}$ & References $[16,24]$ \\
\hline 24 & Gelsenine & NA & 358.19 & $\mathrm{C}_{20} \mathrm{H}_{26} \mathrm{~N}_{2} \mathrm{O}_{4}$ & References $[16,24]$ \\
\hline 25 & 21- Oxokoumine & NA & 320.1 & $\mathrm{C}_{20} \mathrm{H}_{20} \mathrm{~N}_{2} \mathrm{O}_{2}$ & References $[16,24]$ \\
\hline 26 & Furanokoumine & NA & 322.1 & $\mathrm{C}_{20} \mathrm{H}_{22} \mathrm{~N}_{2} \mathrm{O}_{2}$ & References $[16,24]$ \\
\hline 27 & Koumidine & $44,584,550$ & 294.4 & $\mathrm{C}_{19} \mathrm{H}_{22} \mathrm{~N}_{2} \mathrm{O}$ & TCMID, BATMAN-TCM, References $[16,24]$ \\
\hline 28 & Gelebolines A & NA & 320.15 & $\mathrm{C}_{20} \mathrm{H}_{20} \mathrm{~N}_{2} \mathrm{O}_{2}$ & References $[16,24]$ \\
\hline 29 & 19- Z- taberpsychine & $5,321,582$ & 310.4 & $\mathrm{C}_{20} \mathrm{H}_{26} \mathrm{~N}_{2} \mathrm{O}$ & References $[16,24]$ \\
\hline 30 & Nb-Methylgelsedilam & NA & 328.14 & $\mathrm{C}_{18} \mathrm{H}_{20} \mathrm{~N}_{2} \mathrm{O}_{4}$ & References $[16,24]$ \\
\hline 31 & Gelsesyringalidine & $136,704,418$ & 490.5 & $\mathrm{C}_{28} \mathrm{H}_{30} \mathrm{~N}_{2} \mathrm{O}_{6}$ & References $[16,24]$ \\
\hline 32 & 14- Dehydroxygelsefuranidine & $102,417,029$ & 404.5 & $\mathrm{C}_{24} \mathrm{H}_{24} \mathrm{~N}_{2} \mathrm{O}_{4}$ & References $[16,24]$ \\
\hline 33 & Humantenoxenine & NA & 368.17 & $\mathrm{C}_{21} \mathrm{H}_{24} \mathrm{~N}_{2} \mathrm{O}_{4}$ & References $[16,24]$ \\
\hline 34 & Kounaminal & $102,260,292$ & 363.5 & $\mathrm{C}_{22} \mathrm{H}_{25} \mathrm{~N}_{3} \mathrm{O}_{2}$ & References $[16,24]$ \\
\hline 35 & Dehydrokoumidine & $119,077,162$ & 292.4 & $\mathrm{C}_{19} \mathrm{H}_{20} \mathrm{~N}_{2} \mathrm{O}$ & References $[16,24]$ \\
\hline 36 & Koumine & $91,895,267$ & 306.4 & $\mathrm{C}_{20} \mathrm{H}_{22} \mathrm{~N}_{2} \mathrm{O}$ & $\begin{array}{l}\text { TCMID, BATMAN-TCM, TCM-Taiwan, Refer- } \\
\text { ences }[16,24]\end{array}$ \\
\hline 37 & Gelsemine & 279,057 & 322.4 & $\mathrm{C}_{20} \mathrm{H}_{22} \mathrm{~N}_{2} \mathrm{O}_{2}$ & TCMID, BATMAN-TCM, Reference [16] \\
\hline 38 & Elegansamine & $5,317,023$ & 508.6 & $\mathrm{C}_{29} \mathrm{H}_{36} \mathrm{~N}_{2} \mathrm{O}_{6}$ & TCMID, BATMAN-TCM \\
\hline 39 & Ranol & $10,939,065$ & 438.6 & $\mathrm{C}_{26} \mathrm{H}_{46} \mathrm{O}_{5}$ & TCMID, BATMAN-TCM, TCM-Taiwan \\
\hline 40 & 12a-Hudroxyrotenone & 161,176 & 410.4 & $\mathrm{C}_{23} \mathrm{H}_{22} \mathrm{O}_{7}$ & TCMID, TCM-Taiwan \\
\hline
\end{tabular}


Table 1 Information of the active compounds in GEB for network analysis (Continued)

\begin{tabular}{|c|c|c|c|c|c|}
\hline NO & Name & $\begin{array}{l}\text { Compound } \\
\text { CID }\end{array}$ & MW & MF & Source \\
\hline$\overline{41}$ & $\begin{array}{l}\text { 5-methoxy-7-(4-hydroxy-33methoxy phenyl)-1-phenyl-3- } \\
\text { heptanone }\end{array}$ & $26,088,037$ & 342.4 & $\mathrm{C}_{21} \mathrm{H}_{26} \mathrm{O}_{4}$ & TCMID, TCM-Taiwan \\
\hline 42 & 15-Hydroxyhumamtenine & $101,606,434$ & 370.4 & $\mathrm{C}_{21} \mathrm{H}_{26} \mathrm{~N}_{2} \mathrm{O}_{4}$ & TCMID \\
\hline 43 & Humantenine & $44,593,672$ & 354.4 & $\mathrm{C}_{21} \mathrm{H}_{26} \mathrm{~N}_{2} \mathrm{O} 3$ & TCMID, BATMAN-TCM, TCM-Taiwan \\
\hline 44 & Akuammidine $\mathrm{N}$-Oxide & $11,268,654$ & 368.4 & $\mathrm{C}_{21} \mathrm{H}_{24} \mathrm{~N}_{2} \mathrm{O}_{4}$ & TCMID, BATMAN-TCM, TCM-Taiwan \\
\hline 45 & 19-(Z)-Akuammidine & $44,583,830$ & 352.4 & $\mathrm{C}_{21} \mathrm{H}_{24} \mathrm{~N}_{2} \mathrm{O}_{3}$ & TCMID, TCM-Taiwan \\
\hline 46 & Tabersonine & 20,485 & 336.4 & $\mathrm{C}_{21} \mathrm{H}_{24} \mathrm{~N}_{2} \mathrm{O}_{2}$ & TCMID, BATMAN-TCM, TCM-Taiwan \\
\hline 47 & Gelsemicine & $5,462,428$ & 358.4 & $\mathrm{C}_{20} \mathrm{H}_{26} \mathrm{~N}_{2} \mathrm{O}_{4}$ & TCMID, BATMAN-TCM, TCM-Taiwan \\
\hline 48 & 19-(Z)-Taberpsychine & $5,321,582$ & 310.4 & $\mathrm{C}_{20} \mathrm{H}_{26} \mathrm{~N}_{2} \mathrm{O}$ & TCMID, BATMAN-TCM \\
\hline 49 & 19-(S)-Hydroxydihydrokoumine & $5,318,193$ & 324.4 & $\mathrm{C}_{20} \mathrm{H}_{24} \mathrm{~N}_{2} \mathrm{O}_{2}$ & TCMID, TCM-Taiwan \\
\hline 50 & n-desmethoxtrankinidine & $5,316,593$ & 324.4 & $\mathrm{C}_{20} \mathrm{H}_{24} \mathrm{~N}_{2} \mathrm{O}_{2}$ & TCMID \\
\hline 51 & Oxoglaucine & 97,662 & 351.4 & $\mathrm{C}_{20} \mathrm{H}_{17} \mathrm{NO}_{5}$ & TCMID, BATMAN-TCM, TCM-Taiwan \\
\hline 52 & Sempervirine (II) & 168,919 & 272.3 & $\mathrm{C}_{19} \mathrm{H}_{16} \mathrm{~N}_{2}$ & TCMID, TCM-Taiwan \\
\hline 53 & 6-methoxygeniposidic acid & $6,325,623$ & 404.4 & $\mathrm{C}_{17} \mathrm{H}_{24} \mathrm{O}_{11}$ & TCMID \\
\hline 54 & hydroxygenkwanin & $5,318,214$ & 300.3 & $\mathrm{C}_{16} \mathrm{H}_{12} \mathrm{O}_{6}$ & TCMID \\
\hline 55 & dihydromelilotoside & $5,316,728$ & 328.3 & $\mathrm{C}_{15} \mathrm{H}_{20} \mathrm{O}_{8}$ & TCMID \\
\hline 56 & 7-hydroxydihydromatatabiether & $5,318,195$ & 170.4 & $\mathrm{C}_{10} \mathrm{H}_{18} \mathrm{O}_{2}$ & TCMID \\
\hline
\end{tabular}

NA: Not applicable

\section{PPI network of targets for GEB against CRC}

To further identify the core regulatory targets of GEB against CRC, the STRING tool was employed to establish PPI network of the 136 targets. The PPI network of the potential targets of GEB against CRC was shown in Fig. 5A. With the confidence score $>0.7$ was selected, the network of PPI was composed of 118 nodes and 458 edges. The top 20 proteins with higher levels of connectivity were selected as the center targets for GEB against CRC. The center targets, which may play a key role in
GEB against CRC, namely, MAPK3, HSP90AA1, JUN, EGFR, CDK1, TNF, CCND1, ESR1, PRKACA, CCNA2, CDC25C, CDK2, CCNB1, AR, CREBBP, AURKA, CDC25A, CHEK1, BCL2L1, and PIK3CD. Three topological features of these top 20 targets exhibited mean values of degree, node betweenness, and closeness were $20.75,0.05843$ and 0.4442 , respectively.

Protein-protein interaction of 136 potential targets (A) and 7 clusters (B, C, D, E, F, G, and H). Edges represent protein-protein associations, including known

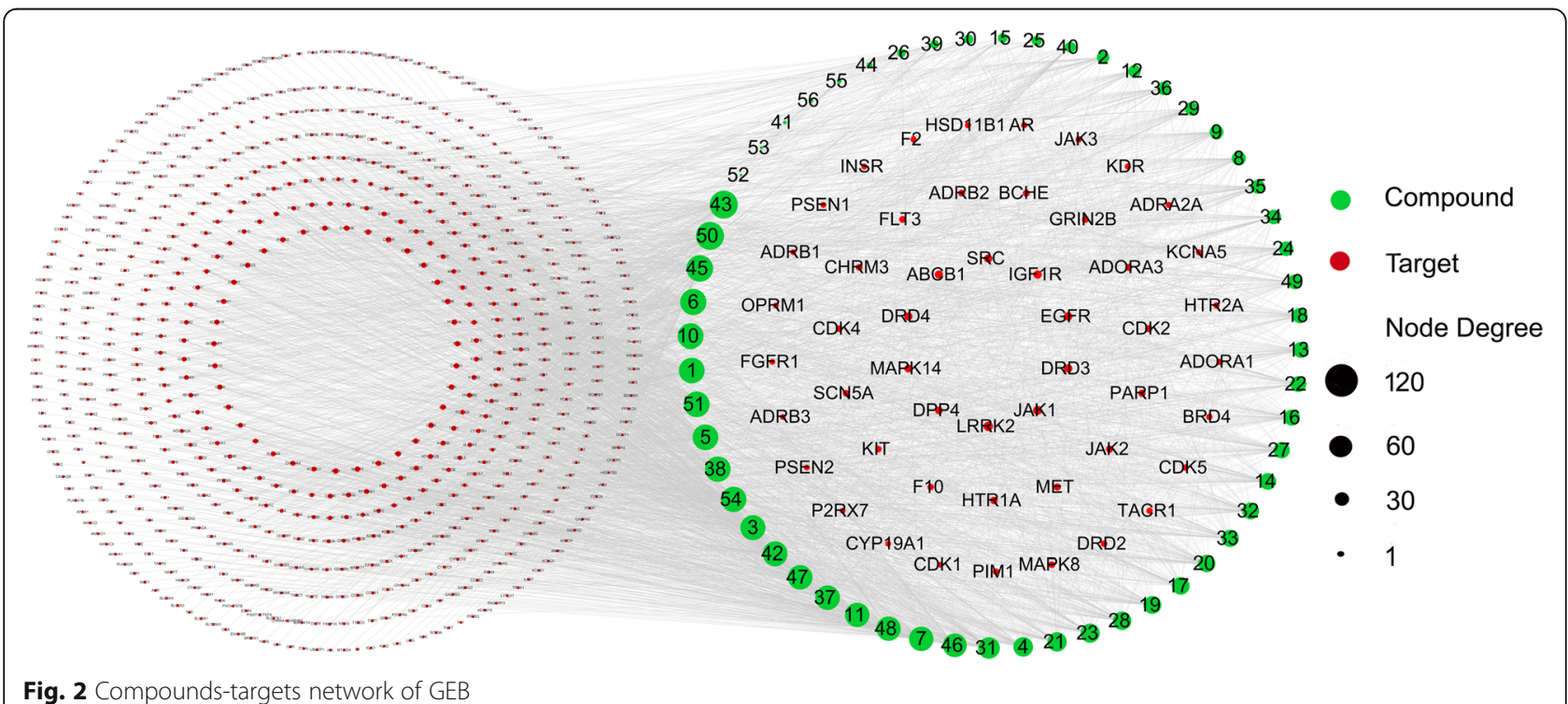

Fig. 2 Compounds-targets network of GEB 
A. GEB target genes

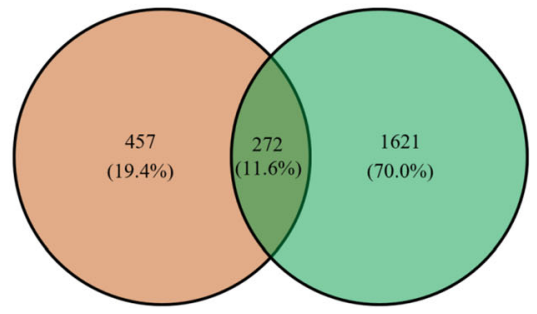

B. Candidate genes TCGA database

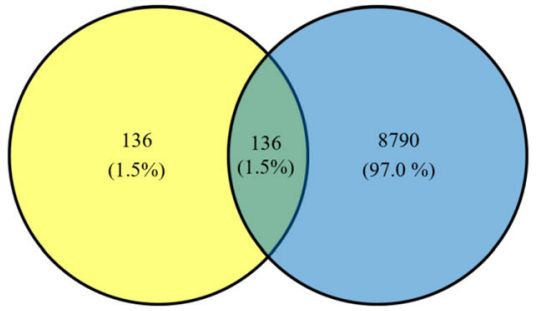

Fig. 3 GEB target genes prediction for CRC treatment

interaction (azure represents curated database evidence, purple represents experimentally determined evidence), predicted interactions (green represents gene neighborhood, red represents gene fusions, and blue represents gene co-occurrence), and others (light green represents text mining, black represents co-expression, and light blue represents protein homology).

\section{Cluster analysis}

MCODE network analysis revealed 7 clusters (Fig. 5B, C, D, E, F, G, and H). The highest scoring cluster, cluster 1, contained 13 nodes and 58 edges, including CCND2, TOP2A, CDK4, TTK, PLK1, CCNA2, CCND1, CCNB1, PLK4, CDK1, CDC25C, CHEK1, and AURKA. Cluster 2 contained 5 nodes (CDK2, CDC25A, CCNE1, WEE1, and CCNE2) and 10 edges. Cluster 3 contained 5 nodes (JUN, ESR1, EGFR, NR3C1, and ESR2) and 8 edges. The detail of other clusters was shown in Table 2.

\section{GO and KEGG pathway enrichment analysis}

To explore the multiple functions of 136 potential antiCRC targets of GEB, GO analysis and KEGG pathway enrichment of the candidate targets were performed. In $\mathrm{GO}$ analysis, the 136 potential target genes were significantly enriched in 70 biological process (BP), 22 cell components (CC), and 24 molecular functions (MF) $($ FDR $<0.01$, Supplementary Table S6). The top 3 BP terms were peptidyl-tyrosine phosphorylation (GO:

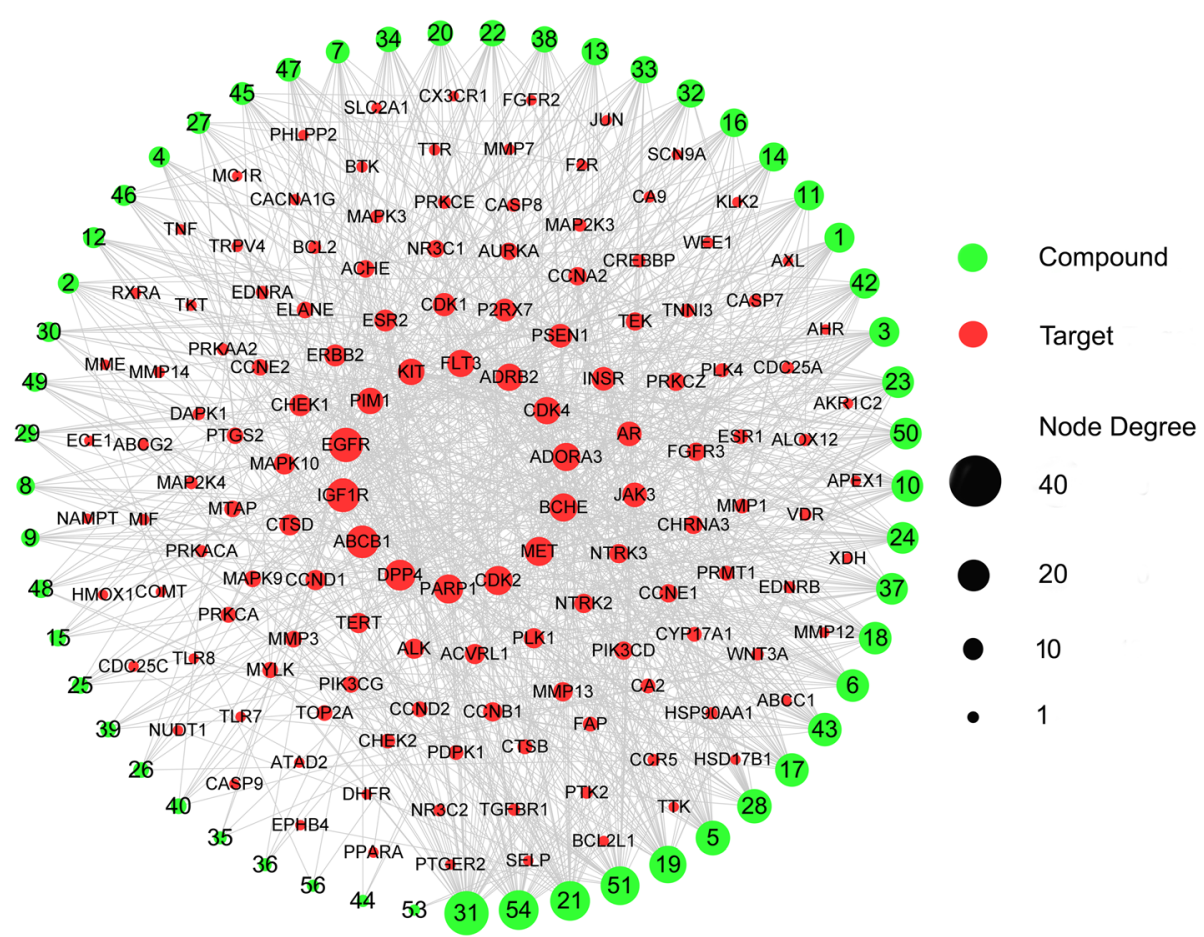

Fig. 4 Compounds-potential targets network of GEB in treatment for CRC 


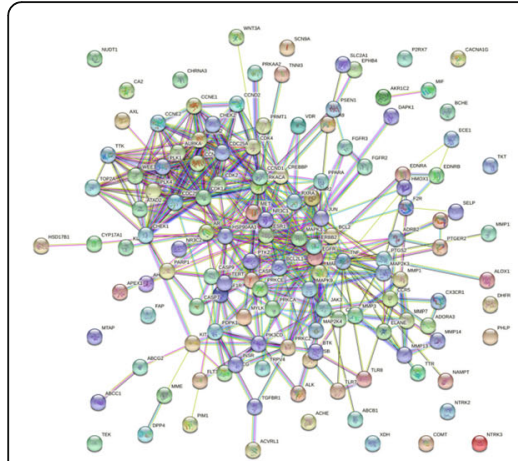

A. PPI of 136 targets

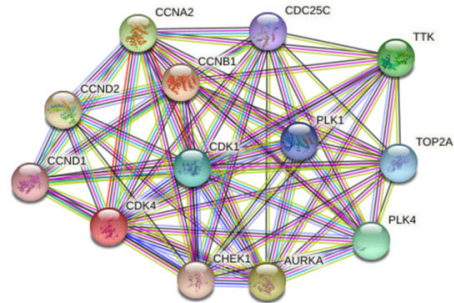

B. Cluster 1

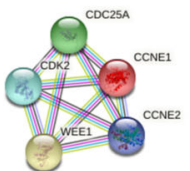

C. Cluster 2

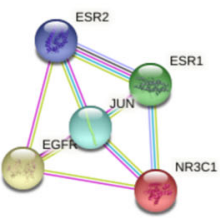

D. Cluster 3

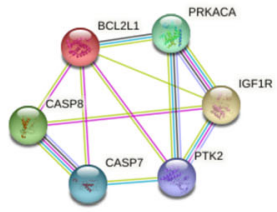

E. Cluster 4

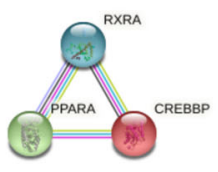

F. Cluster 5

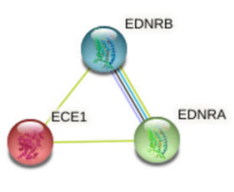

G. Cluster 6

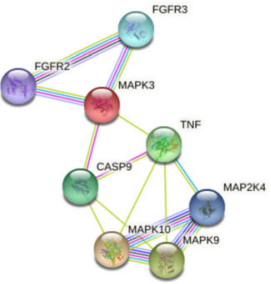

H. Cluster 7
Known interactions

From curated database

$\ominus$ Experimentally determined

Predicted interactions

$\ominus$ Gene neighbour

e-s Gene fusions

e-gene co-occurance

Others

s-T Textmining

$\ominus$ Co-expression

e-Protein holomogy

Fig. 5 Protein-protein interaction network for GEB in treatment for CRC

0018108), protein autophosphorylation (GO: 0006468). The top $3 \mathrm{CC}$ terms included cytosol (GO: 0005829), nucleoplasm (GO:0005654), and cell surface (GO:0009986). The top $3 \mathrm{MF}$ terms were mainly enriched in ATP binding (GO:0005524), transmembrane receptor protein tyrosine kinase activity (GO: 0004714), and protein kinase activity (GO: 0004672). KEGG pathway analysis identified 70 pathways that potentially participate in the anti-CRC of GEB. The top 3 pathways were pathways in cancer (hsa05200), prostate cancer (hsa05215), and PI3K-Akt signaling pathway (hsa04151). The pathway of colorectal cancer (hsa05210) was also remarkably enriched (FDR <
0.01, Supplementary Table S7). Top 10 GO functional categories in $\mathrm{BP}, \mathrm{CC}$, and $\mathrm{MF}$, and 25 remarkable pathways were selected and were presented in Fig. 6.

The GO enrichment analysis of GEB anti-CRC genes (A). The ontology covered 3 domains: biological process, cellular component, and molecular function. The KEGG enrichment analysis of GEB against CRC related genes (B). The abscissa represents the rich factors, the proportion of genes enriched in the according to the pathway, the ordinate represents the pathway, and the color of the circle represents the corrected FDR-value. KEGG- Kyoto Encyclopedia of Genes and Genomes; GO- Gene Ontology.

Table 2 Clusters of the protein-protein interaction (PPI) network

\begin{tabular}{lllll}
\hline Cluster & Score & Nodes & Edges & Gene IDs \\
\hline 1 & 9.667 & 13 & 58 & CCND2, TOP2A, CDK4, TTK, PLK1, CCNA2, CCND1, CCNB1, PLK4, CDK1, CDC25C, CHEK1, AURKA \\
2 & 5 & 5 & 10 & CDK2, CDC25A, CCNE1, WEE1, CCNE2 \\
3 & 4 & 5 & 8 & JUN, ESR1, EGFR, NR3C1, ESR2 \\
4 & 3.2 & 6 & 8 & PTK2, IGF1R, CASP8, PRKACA, CASP7, BCL2L1 \\
5 & 3 & 3 & 3 & PPARA, CREBBP, RXRA \\
6 & 3 & 3 & 3 & EDNRA, ECE1, EDNRB \\
7 & 2.857 & 8 & 10 & MAPK3, MAP 2 K4, CASP9, FGFR3, FGFR2, MAPK9, TNF, MAPK10 \\
\hline
\end{tabular}




\section{The most enriched GO terms}

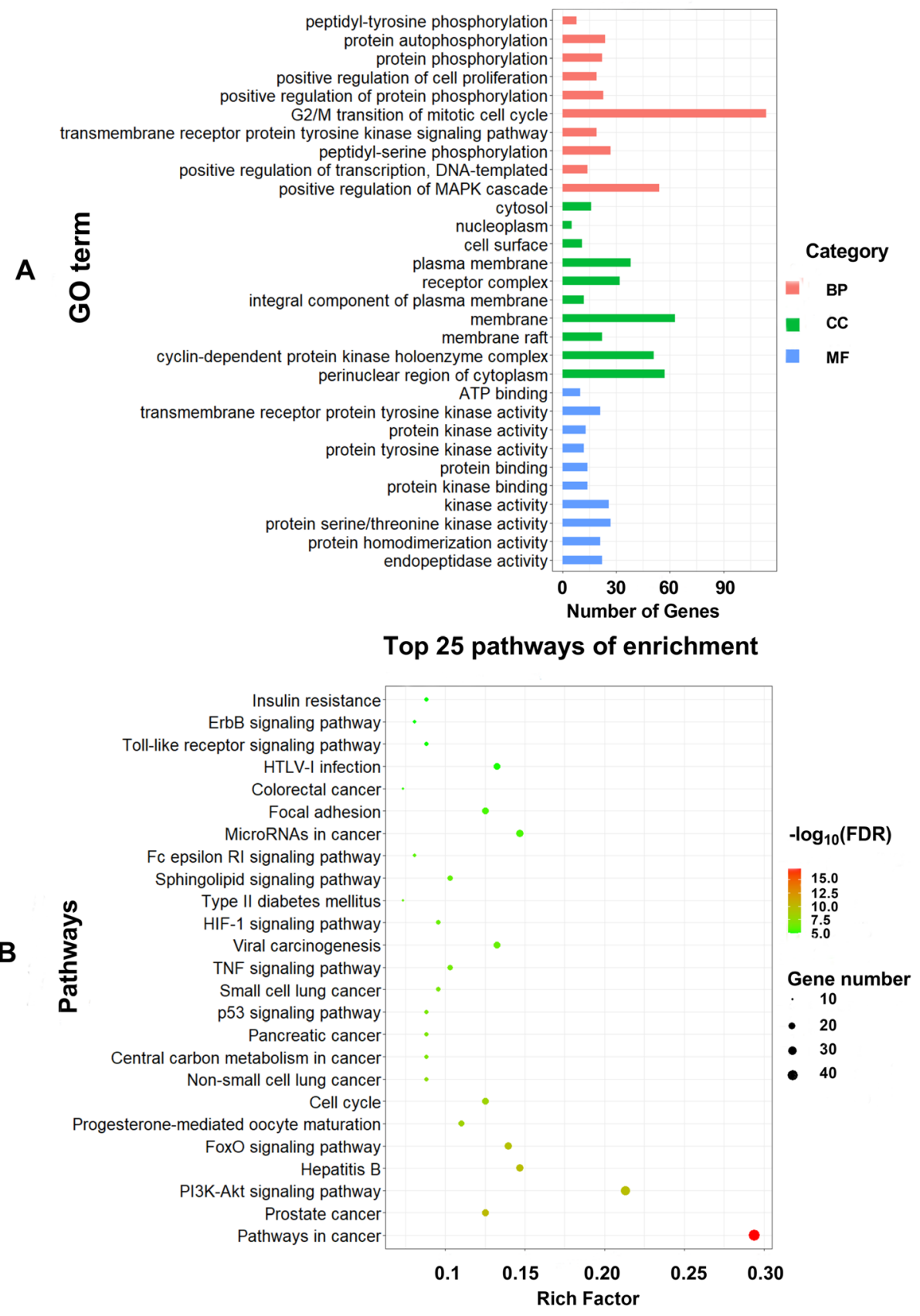

Fig. 6 GO and KEGG pathway enrichment analysis of GEB in treatment for CRC

\section{Compound-target-pathway network construction}

To explain the mechanism of GEB against CRC, a compound-target pathway network was constructed based on the above compounds, targets, and pathways information. As depicted in Fig. 7, the network was composed of 96 nodes (51 compounds, 20 targets, and 25 pathways) and 315 edges. The green circles, red circles, and pink squares represent active compounds, target proteins, and potential pathways involved in process of GEB against CRC, respectively. The top 3 active compounds with the most degrees are gelegamine $\mathrm{E}$, gelsesyringalidine, and humantenine. The top 3 target proteins with the most degrees are EGFR, PIK3CD, and CDK2.

Red circle nodes stand for the potential target genes related to CRC. Green nodes stand for potential active 


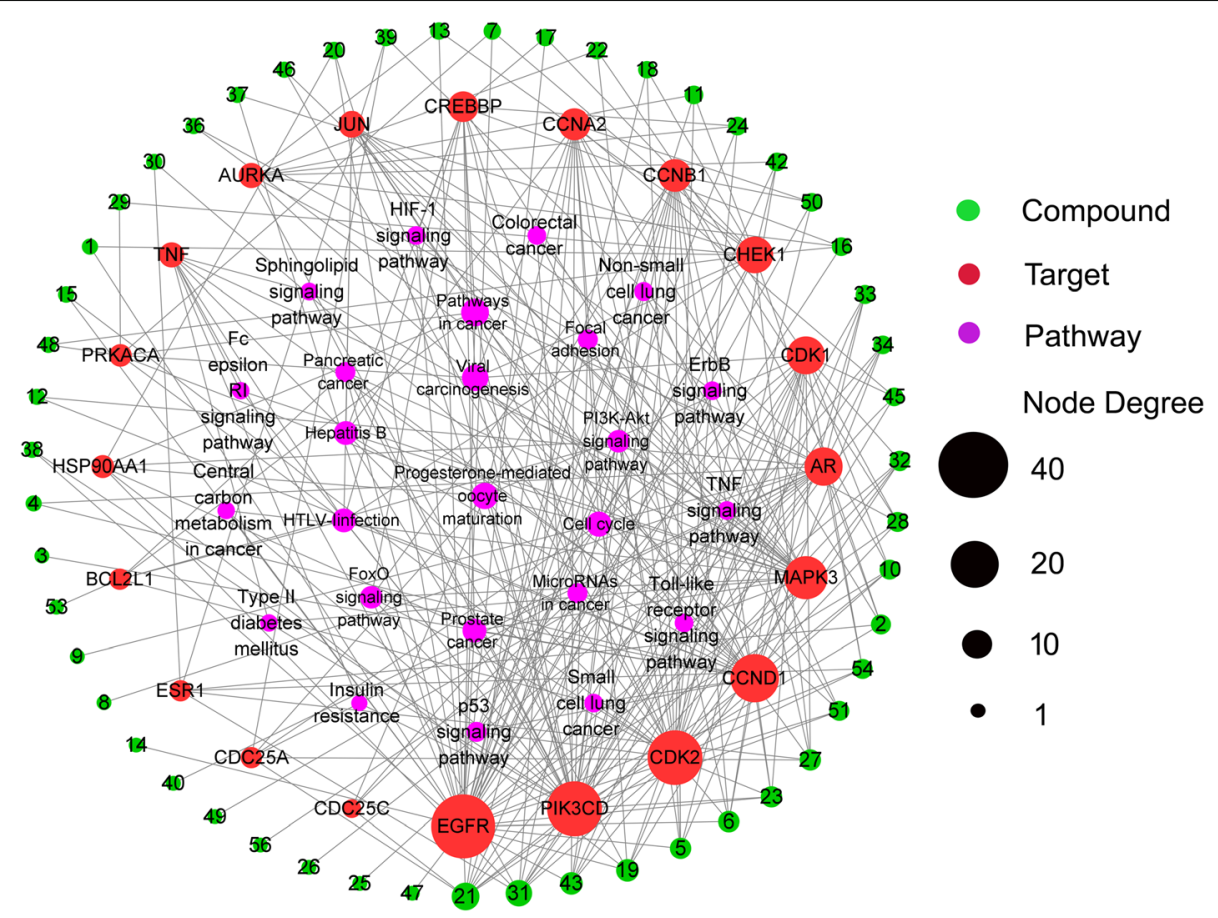

Fig. 7 The potential compounds-targets-pathways network of GEB for treating CRC

compounds in GEB for treating CRC. Pink squares stand for pathways. The size of the node represents the number of the degrees.

\section{Compounds-targets docking}

The interaction between components and targets was further evaluated by applying molecular docking, which will validate the accuracy of the compound-target networks analysis.

Virtual screening using AutoDock Vina was performed to calculate the binding affinity between protein models and 10 potentially active compounds (including gelegamine E, gelsesyringalidine, humantenine, 19 $\alpha$ hydroxygelsamydine, gelsedine, 19-Z-akuammidine, gelegamine $\mathrm{B}$, tabersonine, koumine and gelsemine) obtained from compound-target-pathway network. Six targets (EGFR, PIK3CD, CDK2, CCND1, MAPK3, and AR) and 10 compounds were analyzed by molecular docking (Table 3). The top 4 Compound-target dockings with the lowest binding energy were visualized in Fig. 8. The complex of gelsesyringalidine-CDK2 was stabilized by two H-bond with residues including ASP-86 (3.5 $\AA$ ) and LYS-89 (2.4 $\AA$ ), the complex of gelsesyringalidineMAPK3 was stabilized by five $\mathrm{H}$-bond with residues including LYS-131 (2.1 А, $2.8 \AA)$, TYR-53 (3.2 $)$ and MET-125 (2.4 Å, $2.5 \AA ̊$ ) (Fig. 8A, B). Meanwhile, 19 $\alpha-$ hydroxygelsamydine fixed the binding cavity of CRC target EGFR through one $\mathrm{H}$-bonds with residue including SER-720 (2.6 $\AA$ ), and hydroxygelsamydine-MAPK3 was stabilized by two $\mathrm{H}$-bonds with residues including TYR53(3.1 $\AA$ ) and GLU-50 (2.4 ^) (Fig. 8C, D).

The virtual docking of gelsesyringalidine with CDK2 and MAPK3 was represented by $\mathrm{A}$ and $\mathrm{B}$, respectively. The virtual docking of $19 \alpha$-Hydroxygelsamydine with EGFR and MAPK3 was represented by $\mathrm{C}$ and D, respectively.

\section{Molecular dynamics simulation}

Molecular dynamics simulation provides a significant insight about the stability of protein-ligand complex. As shown in Fig. 9, the best hit molecules after docking, gelsesyringalidine-CDK2 binding complex showed similar RMSD and RMSF value in comparison to primitive ligand complex AJR-CDK2 binding complex. This observation indicates that gelsesyringalidine forms a stable protein-ligand complexe and does not make any considerable conformational change in the protein structure during simulation.

RMSD and RMSF profiles of ligand-protein complex were represented by $A$ and $B$, respectively. ARJ, primitive ligand of CDK2: 2,2' $-\{[6-\{[(4-$ methoxyphenyl)methyl] amino\}-9-(propan-2-yl)-9H-purin-2-yl]azanediyl $\}$ di(ethan-1-ol).

\section{Discussion}

The Chinese name of GEB is "Duan chang cao", which literally means a plant can lead to the intestines broken. On the one hand, it shows that this plant has strong 
Table 3 Virtual docking of ten bioactive compounds of GEB and CRC targets

\begin{tabular}{|c|c|c|c|c|c|c|}
\hline NO & Name & The number of Hydrogen bond & Amino acid residue & Structure & Target & $\begin{array}{l}\text { Binding Energy } \\
\left(\mathrm{kcal} \cdot \mathrm{mol}^{-1}\right)\end{array}$ \\
\hline \multirow[t]{4}{*}{21} & Gelegamine $\mathrm{E}$ & 1 & LYS'745 & & EGFR & -8.0 \\
\hline & & 4 & ARG' 870 & & PIK3CD & -5.3 \\
\hline & & 1 & ASP’86 & & CDK2 & -7.7 \\
\hline & & 2 & ARG 59 & & CCND1 & -6.8 \\
\hline \multirow[t]{3}{*}{31} & Gelsesyringalidine & 4 & ARG' 870/GLU' 873/SER' 878 & & PIK3CD & -2.9 \\
\hline & & 2 & ASP'86/LYS 89 & & CDK2 & -10.1 \\
\hline & & 5 & LYS 131/MET'125/TYR`53 & & MAPK3 & -9.5 \\
\hline \multirow[t]{2}{*}{43} & Humantenine & NA & NA & & EGFR & -8.3 \\
\hline & & 1 & LYS 89 & & CDK2 & -5.7 \\
\hline \multirow[t]{2}{*}{19} & 19a-Hydroxygelsamydine & 1 & $E R ` 720$ & $0_{0}^{H}$ & EGFR & -9.5 \\
\hline & & 2 & TYR`53/GLU`50 & & MAPK3 & -9.7 \\
\hline 5 & Gelsedine & 3 & ARG`59/LYS`114 & & CCND1 & -6.7 \\
\hline 6 & 19 - Z- akuammidine & 1 & ARG 52 & & CCND1 & -6.6 \\
\hline 23 & Gelegamine B & 4 & ARG' 870 & & PIK3CD & -6.3 \\
\hline 46 & Tabersonine & 1 & LYS`131 & & MAPK3 & -9.2 \\
\hline
\end{tabular}


Table 3 Virtual docking of ten bioactive compounds of GEB and CRC targets (Continued)

\begin{tabular}{|c|c|c|c|c|c|c|}
\hline NO & Name & The number of Hydrogen bond & Amino acid residue & Structure & Target & 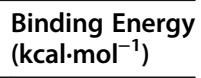 \\
\hline \multirow[t]{2}{*}{36} & Koumine & NA & NA & & $A R$ & -9.0 \\
\hline & & 1 & SER`170 & & MAPK3 & -7.9 \\
\hline 37 & Gelsemine & 1 & CYS'797 & & EGFR & -8.2 \\
\hline
\end{tabular}

NA Not applicable

toxicity, on the other hand, it may imply a potential target site related to the digestive tract. In China, GEB is used in folk for the treatment of different diseases, including cancer [18]. Consistently, GEB is thought to have anti-cancer properties and exhibit pharmaceutical potential. However, its clinical use is hampered by its toxicity. The prediction of the toxicological hazard when administered orally from the molecular structure of compounds of GEB were evaluated by Toxtree (Table S8). Clinical application of GEB has not been officially approved and is now only used in folk. Despite the high toxicity of GEB and its crude alkaloidal extraction, some monomeric alkaloids of GEB are relatively low in toxicity [16]. For instance, koumine $(4.8 \mathrm{mg} / \mathrm{kg}$, intraperitoneally) exhibited significant antitumor activity on mice bearing solid tumor [38]. Whereas, LD50 of koumine is $100 \mathrm{mg} /$ $\mathrm{kg}$ (mice, intraperitoneally) [16], demonstrating a high therapeutic index in the treatment of cancer. Hence, GEB may provide a promising number of molecules with proven cytotoxic and apoptogenic activities against CRC, GEB and its alkaloidal components have been abstracting increasing attention for development of antineoplastic drugs $[22,39,40]$.

However, the multiple targets, pathways, and mechanisms of its antitumor effect remain unclear. Network pharmacology has been widely adopted by many studies to investigate the potential activity, targets, and pathways of medicinal plants, herb pairs, or herbal formulas with

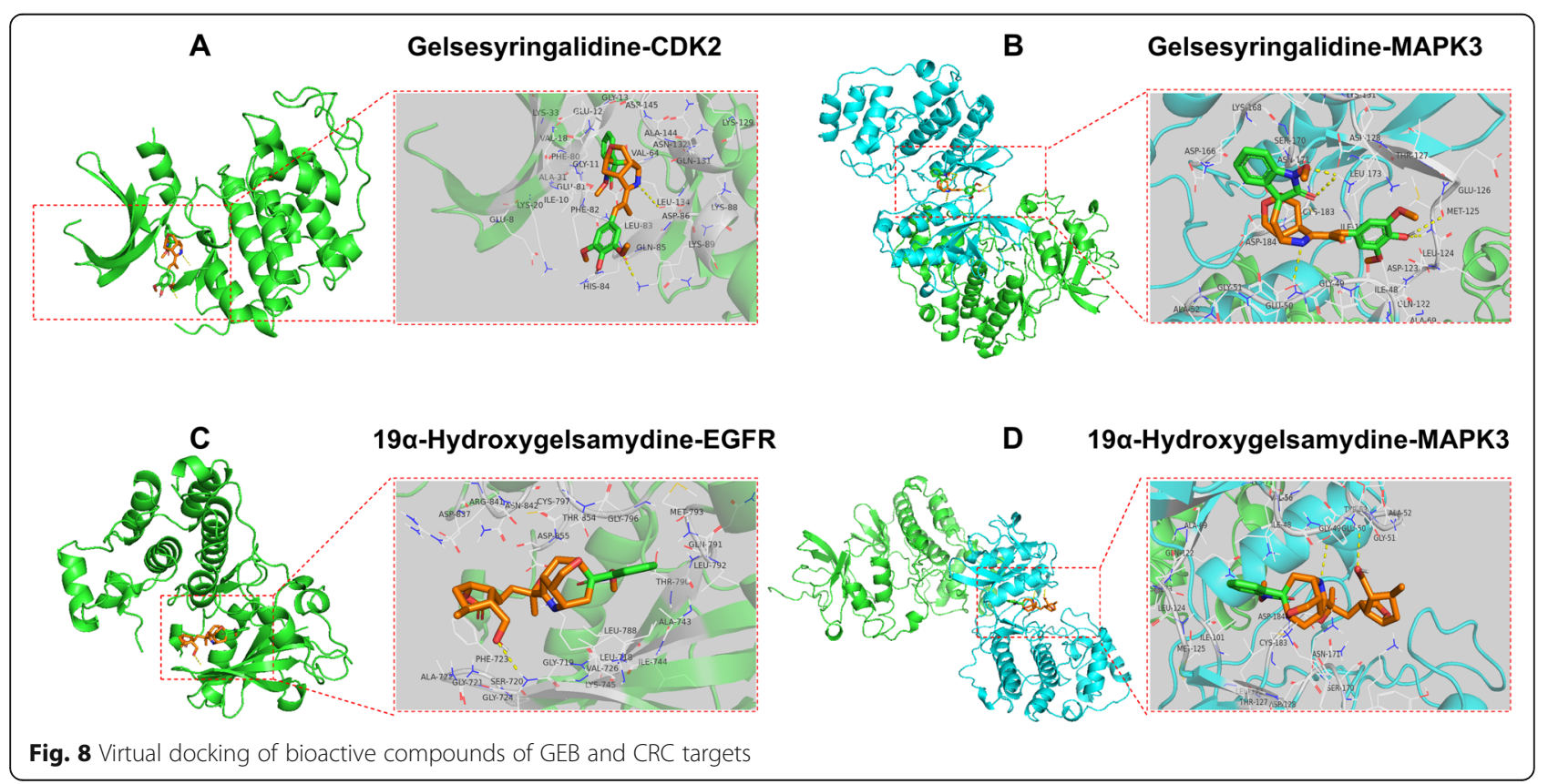




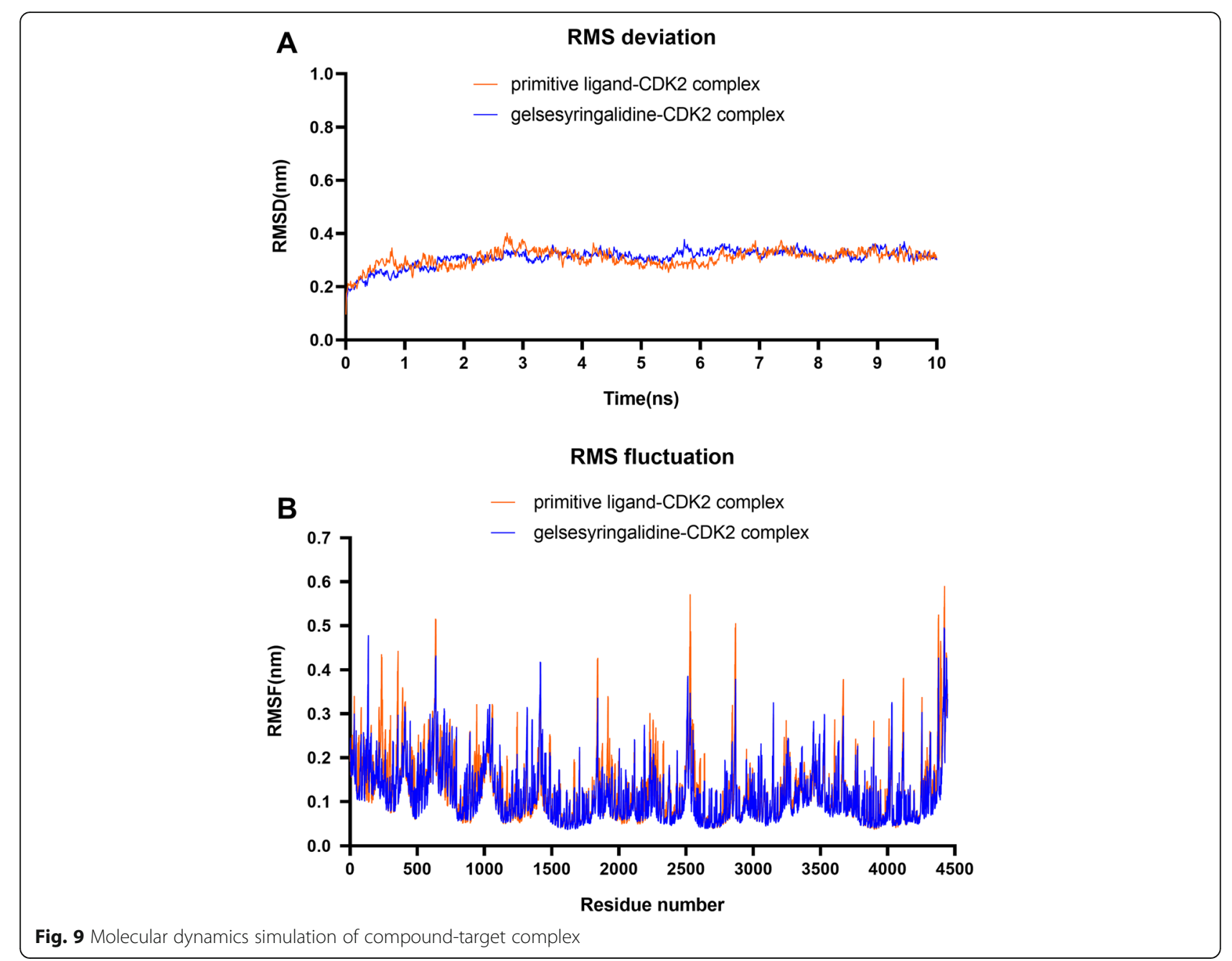

complex ingredients $[41,42]$. Thus, network pharmacology provides a novel opportunity for us to investigate potentially pharmacological and molecular mechanisms of GEB against CRC in this study. To the best of our knowledge, this is the first study applying network pharmacology analyses to reveal the pharmacological mechanisms of GEB for treating CRC.

In the present study, a total of 56 compounds of GEB were included in the network pharmacology-based analysis, 53 active compounds were identified with 136 potential targets related to CRC. Many targets were discovered to be hit by multiple compounds. For example, the top 3 targets, EGFR, IGF1R, and ABCB1 were modulated by more than 20 ingredients. Also, ingredients of GEB such as gelsesyringalidine, Gelegamine E, and hydroxygenkwanin can regulate more than 30 targets. This fact indicated that the bioactive ingredients of GEB might regulate multiple targets and can affect these targets synergistically. Therefore, active ingredients of GEB have therapeutic effects not only on CRC but also on other diseases, which virtually confirmed the nature of multicomponent, multi-target, and multi-disease of plant medicine. Hence, we could not only acquire valuable information on the relationship between active ingredients and its potential targets but also discover the other potential effects of GEB from the network pharmacology-based analysis.

PPI analysis of 136 targets revealed that the top 20 of center target genes, were MAPK3, HSP90AA1, JUN, EGFR, CDK1, TNF, CCND1, ESR1, PRKACA, CCNA2, CDC25C, CDK2, CCNB1, AR, CREBBP, AURKA, CDC25A, CHEK1, BCL2L1, and PIK3CD, which were likely to be crucial targets for GEB in treating CRC. GEB may exert its therapeutic effect against CRC by regulating these specific protein targets. Consider MAPK3, HSP90AA1, JUN, EGFR, CDK1. MAPK3 was simultaneously targeted by 4 active chemicals: $19 \alpha-$ hydroxygelsamydine, gelsesyringalidine, koumine, and tabersonine. Mitogen-activated protein kinase 3 (MAPK3) belongs to the protein kinase superfamily and 
catalyzes the concomitant phosphorylation of a threonine and a tyrosine residue in the MAP kinase p38. It has been confirmed to be a potential therapeutic target for different kinds of human cancers, including CRC [43-47]. A previous study revealed that phosphorylated AMP-activated protein kinase (AMPK) expression in $\mathrm{CRC}$ was associated with superior prognosis among pMAPK3 positive cases, indicating a possible interaction between the AMPK and MAPK pathways influencing tumor behavior [46]. HSP90AA1, a heat shock protein HSP 90-alpha, promotes the maturation, structural maintenance, and proper regulation of particular target proteins involved in signal transduction and cell cycle control. HSP90AA1 is tightly related to gastrointestinal cancers, such as esophageal, gastric, and colon cancers, and can be predictive biomarkers for these cancers [48]. As for JUN, it is known as transcription factor AP-1, is involved in activated KRAS-mediated transcriptional activation of USP2 8 by binding to the USP2 8 promoter in CRC cells. Its mutation in the promoter region is associated with increased CRC risk by elevating promoter activity [49]. JUN plays a key role in regulating and promoting the signaling pathways related to carcinogenesis, cell proliferation, metabolism, migration, apoptosis, and survival [50-52]. EGFR, the ErbB family of related cell membrane receptors, is a receptor tyrosine kinase binding ligand of the EGF family. EGFR family is associated with anti-apoptosis, proliferation, metastasis, and drug resistance in CRC, making this pathway a particularly compelling target for drug design [53]. Notably, EGFR was predicted to correlate with the most active ingredients (28 of 53) of GEB, suggesting that these active components of GEB may exert therapeutic effects synergistically via regulating EGFR expression or its function. CDK1 plays a crucial role in controlling the eukaryotic cell cycle by modulating the centrosome cycle as well as the mitotic onset. It promotes G2-M transition and regulates G1 progress and G1-S transition via association with multiple interphase cyclins. Accordingly, expression of CDK1 has been demonstrated to be enhanced in CRC $[54,55]$. Though the study on the molecular mechanism of anti-cancer action of GEB is limited, fortunately, consistent with the prediction of our study, a previous research has shown that koumine, the most abundant ingredient in alkaloidal components of GEB, suppressed hepatocellular carcinoma cell proliferation via MAPK signaling pathway [56]. Furthermore, the compoundstargets docking analysis results also demonstrated that there was good affinity between MAPK3 and several compounds of GEB including koumine, confirming the role of MAPK3 as one of the key targets in the anticancer effect of GEB. On the whole, our result suggested that active compounds of GEB might produce anticancer effects by interacting with these key targets.
In order to better understand the multiple mechanisms of GEB against CRC from a systematic point of view, we performed a GO enrichment analysis of the 136 selected targets, consisting of the biological processes, molecular functions, and cellular components. Functional enrichment analysis revealed the overrepresented GO terms and their functional domains. The top $10 \mathrm{GO}$ functional categories were shown in Fig. 6 , these demonstrated that GEB may produce its effect by involving in the above biological processes, molecular functions, and cellular components. Based on GO enrichment analysis, BP terms enriched by target genes were mainly concentrated in response to various phosphorylation (GO:0018108: peptidyl-tyrosine phosphorylation, GO:0046777: protein autophosphorylation, GO: 0006468: protein phosphorylation, GO:0001934: positive regulation of protein phosphorylation, and GO:0018105: peptidyl-serine phosphorylation). Protein phosphorylation is a pivotal cellular regulatory mechanism as many enzymes and receptors are activated/deactivated by phosphorylation, which play a key role in the control of biological processes such as proliferation, differentiation and apoptosis [57]. Positive regulation of cell proliferation (GO:0008284) and MAPK cascade (GO:0043410) are also important in colorectal tumorigenesis [58]. Correspondingly, MF terms were strongly correlated with different kinases activity, such as transmembrane receptor protein tyrosine kinase activity (GO:0004714), protein kinase activity (GO:0004672), and protein tyrosine kinase activity (GO:0004713). The functional enrichment analysis implied that GEB might exhibit its anti-CRC effect by regulating of transcription, such as the different kinases activity, resulting in phosphorylation change in cell signaling pathway.

KEGG pathway enrichment analysis revealed that the 136 target proteins were significantly enriched in 70 related signaling pathways. In light of the results of these well-known cancer-related pathway enrichment, we believe GEB can simultaneously target multiple pathways pathways. Among 70 signaling pathways we obtained, colorectal cancer(hsa05210) is the most crucial one that exerts regulatory effect on the process of genetic stability, proliferation, apoptosis, and survival of CRC cells. Furthermore, we found that GEB may exert a therapeutic effect against CRC through other multiple signaling pathways. For instance, FoxO pathway(hsa04068) are involved in cell cycle regulation and proliferation process, activation of FoxO signaling pathway can induce apoptosis effect on human CRC [59]. The activation of the PI3K/AKT pathway(hsa04151) is known to have an important role in the development and progression of CRC, PI3K/AKT signaling leads to reduced apoptosis, stimulates cell growth and increases proliferation [60]. Tumor protein p53 as a well-known transcription 
factor and tumor suppresser, regulates the expression of a wide variety of genes involved in apoptosis, growth arrest, or senescence in response to genotoxic or cellular stress. Hence, inactivation of the p53 pathway is often observed in CRC [61, 62]. MicroRNAs in cancer (hsa05206) participate in tumorigenesis, progression, invasion, and drug resistance in different cancers, including CRC [63]. Chronic inflammation is one of the characteristics of CRC. Tumor necrosis factor alpha $(\mathrm{TNF}-\alpha)$ mediates the inflammatory response, which can activate signal transducer and activator of transcription 3 (STAT3), nuclear factor and kappa-B (NF-kB), resulting in progression of CRC [64]. Yuan reported that koumine could promote ROS production to suppress hepatocellular carcinoma cell proliferation Via NFkappaB signaling and attenuate lipopolysaccaridestimulated inflammation in RAW264.7 macrophages, coincidentally associated with inhibition of NF-kappaB pathways $[56,65]$. These findings may patially support our prediction on KEGG pathway enrichment analysis. To validate prediction of these pathways, microarray technology like Affymetrix GeneChip, may be employed to analysis target gene expression after the treatment of GEB or its key ingredients in future pathway study. Meanwhile, we also found that some signaling pathways significantly enriched by targets were closely related to other cancers, indicating that GEB might exert effects on various malignant tumors, like prostate cancer, lung cancer, and pancreatic cancer.

Total of 53 active compounds were identified by the network pharmacology-based approach. According to Compound-target-pathway network analysis, gelegamine $\mathrm{E}$, gelsesyringalidine, and humantenine were among top 3 key ingredients with the highest degrees in targets. However, they are not the most abundant ingredients in GEB. Hereto, there is no report regarding their pharmacological activities because of their accessibility. With the help of our previous established method of $\mathrm{pH}$-zonerefining counter-current chromatography [66], more monomers from GEB are hopefully available and their anti-CRC effect will be identified. As the most abundant monomer of GEB, Koumine has been attracting much attention in recent decade. Encouragingly, consistent with our prediction, koumine was proved to induce apoptosis of the human colon adenocarcinoma LoVo and SW480 cells $[40,67]$, as well as human hepatoma Bel7402 and H22 cells [38]. In vivo study, koumine also exhibited anticancer effect that comparable with 5fluorouracil in the model of mice bearing the hepatoma cancer [67]. Moreover, koumine is relatively low in toxicity compared with other components, like gelsemine $[16,68]$. Hence, it is promising to discover more novel antineoplastic monomers in GBE with low toxicity based on network pharmacology analysis.

\section{Conclusion}

In this study, based on network pharmacology analysis, we obtained 53 active compounds from GEB and predicted 20 potential center targets for GEB in the treatment of CRC, suggesting that GEB was an herbal medicine with multicomponent, multiple targets, and multiple pathways. The network analysis revealed that GEB may exert its therapeutic effects against CRC by modulating certain distinct targets, such as MAPK3, HSP90AA1, JUN, EGFR, CDK1, TNF CCND1, ESR1, PRKACA, and CCNA2. The GO analysis of these targets demonstrated that the compounds of GEB likely produced pharmacological effects against CRC mainly by influencing different biological processes, like regulation of peptidyl-tyrosine phosphorylation, protein autophosphorylation, and protein phosphorylation. Meanwhile, the KEGG pathway analysis in the present study disclosed that GEB probably exerted its pharmacological action via simultaneously regulating different signaling pathways related to CRC, such as colorectal cancer, pathways in cancer, FoxO signaling pathway, and PI3K-AKT signaling pathway.

To summarize, the present study is the first one that undertakes a network pharmacology- based analysis to explore the potential pharmacological and molecular mechanism of GEB in CRC treatment from a systematic point of view. The results indicated that GEB could be a promising agent in the treatment for CRC with multiple components, targets, and pathways. Our study also provides a theoretical basis for the further development of GEB in the future. However, as this study was based on data mining and data analysis, and there is limited study on the GEB against CRC, more validated experiments are warranted to verify our prediction.

\section{Abbreviations \\ CRC: Colorectal cancer; GEB: Gelsemium elegans Benth; TCM: Traditional Chinese Medicine; TCMID: Traditional Chinese Medicines Integrated Database; BATMAN-TCM: Tool for Molecular mechanism of Traditional Chinese Medicine; DAVID: Database for annotation, visualization and integrated discovery; GO: Gene Ontology; KEGG: Kyoto Encyclopedia of Genes and Genome; TCGA: Cancer Genome Atlas; PPI: Protein-Protein Interaction; MCODE: Molecular Complex Detection; FDR: False Discovery Rate; BP: Biological process; CC: Cellular component; MF: Molecular function; NPTS: Number of grid points in the three dimensions; MAPK3: Mitogen- activated protein kinase 3; AMPK: Phosphorylated AMP-activated protein kinase}

\section{Supplementary Information}

The online version contains supplementary material available at https://doi. org/10.1186/s12906-021-03273-7.

\section{Additional file 1.}

\section{Acknowledgments}

The authors thank Professor Youxiong Que. from Fujian Agriculture and Forestry University for professional advice and guidance on the bioinformatics of the manuscript. 


\section{Authors' contributions}

$\mathrm{QHQ}$ and $\mathrm{CY}$ conceived,designed the experiments, revised and verified the manuscript; QWC and CMH performed the research, analyzed the data, and drafted the manuscript. YL and ZBQ prepared all the figures; ZZC and LM B contributed to the tables and software application, respectively. All authors have read and approved the manuscript.

\section{Funding}

This study was supported by the Joint Funds for the innovation of science and Technology, Fujian province (No. 2019Y9051, 2019Y9040), Wu Jieping Medical Foundation (No. 320.6750.2020-04-47) and the Youth Project of Health Department of Fujian Provice (2020GGB024). These funds covered fee of data collection, article publishing, travel and living expenses, and had no role in the design of the study, analysis and interpretation of data and in writing the manuscript.

\section{Availability of data and materials}

All data generated or analysed during this study are included in this published article and its supplementary information files.

\section{Declarations}

\section{Ethics approval and consent to participate}

Not applicable.

\section{Consent for publication}

Not applicable.

\section{Competing interests}

The authors report no conflicts of interest in this work.

\section{Author details}

'Department of Pharmacy, Fujian Medical University Union Hospital, 29 Xin Quan Rd, Gulou, Fuzhou 350001, Fujian, People's Republic of China. ${ }^{2}$ Department of Pharmacy, Fujian Cancer Hospital \& Fujian Medical University Cancer Hospital, Fuzhou, China. ${ }^{3}$ College of Pharmacy, Fujian Medical University, Fuzhou 350004, People's Republic of China.

Received: 14 December 2020 Accepted: 11 March 2021

\section{Published online: 20 March 2021}

\section{References}

1. Siegel RL, Miller KD, Goding Sauer A, Fedewa SA, Butterly LF, Anderson JC, et al. Colorectal cancer statistics, 2020. CA Cancer J Clin. 2020;70(3):145-64 https://doi.org/10.3322/caac.21601.

2. American Cancer Society: Key statistics for colorectal cancer. https://www.ca ncer.org/cancer/colon-rectal-cancer/about/key-statistics.html. Accessed 2 Dec 2020. (2020)

3. GLOBOCAN: GLOBOCAN 2020: New Global Cancer Data. https://www. uiccorg/news/globocan-2020-new-global-cancer-data. Accessed 26 Jan 2021. (2020)

4. Arnold M, Sierra MS, Laversanne M, Soerjomataram I, Jemal A, Bray F. Global patterns and trends in colorectal cancer incidence and mortality. Gut. 2017; 66(4):683-91. https://doi.org/10.1136/gutjnl-2015-310912.

5. McQuade RM, Stojanovska V, Bornstein JC, Nurgali K. Colorectal cancer chemotherapy: the evolution of treatment and new approaches. Curr Med Chem. 2017;24(15):1537-57. https://doi.org/10.2174/09298673246661701111 52436.

6. Faugeras L, Dili A, Druez A, Krug B, Decoster C, D'Hondt L. Treatment options for metastatic colorectal cancer in patients with liver dysfunction due to malignancy. Crit Rev Oncol Hematol. 2017;115:59-66. https://doi. org/10.1016/j.critrevonc.2017.03.029.

7. Vassos N, Piso P. Metastatic colorectal cancer to the peritoneum: current treatment options. Curr Treat Options Oncol. 2018;19(10):49. https://doi. org/10.1007/s11864-018-0563-8.

8. American Society Of Clinical Oncology: Colorectal Cancer: Statistics. https:// www.cancer.net/cancer-types/colorectal-cancer/statistics. Accessed 2 Dec 2020. (2020)

9. Liu N, Wu C, Jia R, Cai G, Wang Y, Zhou L, et al. Traditional Chinese medicine combined with chemotherapy and cetuximab or bevacizumab for metastatic colorectal cancer: a randomized, double-blind, placebo- controlled clinical trial. Front Pharmacol. 2020;11:478. https://doi.org/10.33 89/fphar.2020.00478

10. Benarba B, Pandiella A. Colorectal cancer and medicinal plants: principle findings from recent studies. Biomed Pharmacother. 2018;107:408-23. https://doi.org/10.1016/j.biopha.2018.08.006.

11. Wang Y, Liu P, Fang Y, Tian J, Li S, Xu J, et al. The effect of long-term traditional Chinese medicine treatment on survival time of colorectal cancer based on propensity score matching: a retrospective cohort study. Evid Based Complement Alternat Med. 2020;2020:7023420.

12. Fei B, Dai W, Zhao S. Efficacy, safety, and cost of therapy of the traditional Chinese medicine, catalpol, in patients following surgical resection for locally advanced colon cancer. Med Sci Monit. 2018;24:3184-92. https://doi. org/10.12659/MSM.907569.

13. Ling CQ, Fan J, Lin HS, Shen F, Xu ZY, Lin LZ, et al. Clinical practice guidelines for the treatment of primary liver cancer with integrative traditional Chinese and western medicine. J Integr Med. 2018;16(4):236-48. https://doi.org/10.1016/j.joim.2018.05.002.

14. Li W, Li C, Zheng H, Chen G, Hua B. Therapeutic targets of traditional Chinese medicine for colorectal cancer. J Tradit Chin Med. 2016;36(2):243-9. https://doi.org/10.1016/s0254-6272(16)30034-6.

15. Dutt V, Thakur S, Dhar VJ, Sharma A. The genus Gelsemium: an update Pharmacogn Rev. 2010;4(8):185-94. https://doi.org/10.4103/0973-7847. 70916.

16. Jin GL, Su YP, Liu M, Xu Y, Yang J, Liao KJ, et al. Medicinal plants of the genus Gelsemium (Gelsemiaceae, Gentianales)--a review of their phytochemistry, pharmacology, toxicology and traditional use. J Ethnopharmacol. 2014;152(1):33-52. https://doi.org/10.1016/j.jep.2014.01.003.

17. Yue R, Jin G, Wei S, Huang H, Su L, Zhang C, et al. Immunoregulatory effect of koumine on nonalcoholic fatty liver disease rats. J Immunol Res. 2019; 2019:8325102

18. Yang K, Huang J, Wu Y, Chen J. Report of 8 cases with primary cancer survived for more than 2 years treated with Gelsemium elegans Benth. J Guangxi Coll. 1981;3:66-8.

19. Li D, Yang Y, Wang Y, Liao H, Lin M, Wu SS. Investigation on folk application of Gelsemium elegans Benth. And detoxification use of different animal blood. J Liaoning Univ TCM. 2016;18(11):23-6.

20. Huang JS, Y P, Yu C, X Y Y, Yang J. Cytotoxic effects of alkaloidal compounds from Gelsemium elegans Benth on the tumor cells of digestive system in vitro. J Strait Phar. 2010;22(3):197-200.

21. Lin MB, Chen L, Wu SS. Effect and mechanism of total alkaloids of Gelsmium elegans on human colonic carcinoma cells proliferation and apoptosis. Chin J Exp Tradit Med Formul. 2018;04:149-53.

22. Wang $W$, Tan $X$, Zhang $P$, Yang Y, Huang Z, Li D, et al. Study on inhibitory effects of total alkaloids of Gelsemium elegans on the proliferation and angiogenesis of human colon cancer cells. Chin Phar. 2020;31(8):957-62

23. Zhao S, lyengar R. Systems pharmacology: network analysis to identify multiscale mechanisms of drug action. Annu Rev Pharmacol Toxicol. 2012 52(1):505-21. https://doi.org/10.1146/annurev-pharmtox-010611-134520.

24. Wu XM, Wu CF. Network pharmacology: a new approach to unveiling traditional Chinese medicine. Chin J Nat Med. 2015;13(1):1-2. https://doi. org/10.1016/S1875-5364(15)60001-2.

25. Zhao YT, Wu SP, Hu CL, J K, Zhao M. Reviews on chemical compositions and pharmacological effect of Gelsemium elegans. Chin J Exp Tradit Med Formul. 2019;25:200-10.

26. Huang L, Xie D, Yu Y, Liu H, Shi Y, Shi T, et al. TCMID 2.0: a comprehensive resource for TCM. Nucleic Acids Res. 2018;46(D1):D1117-D20. https://doi. org/10.1093/nar/gkx1028.

27. Liu Z, Guo F, Wang Y, Li C, Zhang X, Li H, et al. BATMAN-TCM: a bioinformatics analysis tool for molecular mechanism of traditional Chinese medicine. Sci Rep. 2016;6(1):21146. https://doi.org/10.1038/srep21146.

28. Chen CY. TCM database@Taiwan: the world's largest traditional Chinese medicine database for drug screening in silico. PLoS One. 2011;6(1):e15939. https://doi.org/10.1371/journal.pone.0015939.

29. Daina A, Michielin O, Zoete V. SwissADME: a free web tool to evaluate pharmacokinetics, drug-likeness and medicinal chemistry friendliness of small molecules. Sci Rep. 2017;7(1):42717. https://doi. org/10.1038/srep42717.

30. Daina A, Michielin O, Zoete V. SwissTargetPrediction: updated data and new features for efficient prediction of protein targets of small molecules. Nucleic Acids Res. 2019;47(W1):W357-W64. https://doi.org/1 0.1093/nar/gkz382. 
31. Stelzer G, Rosen N, Plaschkes I, Zimmerman S, Twik M, Fishilevich S, et al. The GeneCards suite: from gene data mining to disease genome aequence analyses. Curr Protoc Bioinformatics. 2016;54:1.30.1-1.30.33.

32. Szklarczyk D, Morris JH, Cook H, Kuhn M, Wyder S, Simonovic M, et al. The STRING database in 2017: quality-controlled protein-protein association networks, made broadly accessible. Nucleic Acids Res. 2017;45(D1):D362-D8. https://doi.org/10.1093/nar/gkw937.

33. Bader GD, Hogue CW. An automated method for finding molecular complexes in large protein interaction networks. BMC Bioinformatics. 2003; 4(1):2. https://doi.org/10.1186/1471-2105-4-2.

34. Fang E, Zhang X. Identification of breast cancer hub genes and analysis of prognostic values using integrated bioinformatics analysis. Cancer Biomark. 2017;21(1):373-81. https://doi.org/10.3233/CBM-170550.

35. Dennis G Jr, Sherman BT, Hosack DA, Yang J, Gao W, Lane HC, et al. DAVID: database for annotation, visualization, and integrated discovery. Genome Biol. 2003;4(5):P3. https://doi.org/10.1186/gb-2003-4-9-r60.

36. Kohl M, Wiese S, Warscheid B. Cytoscape: software for visualization and analysis of biological networks. Methods Mol Biol. 2011;696:291-303. https:// doi.org/10.1007/978-1-60761-987-1_18.

37. Gu S, Xue Y, Gao Y, Shen S, Zhang Y, Chen K, et al. Mechanisms of indigo naturalis on treating ulcerative colitis explored by GEO gene chips combined with network pharmacology and molecular docking. Sci Rep. 2020;10(1):15204. https://doi.org/10.1038/s41598-020-71030-w.

38. Wu RD, Qin R, Cai J, Chi DB. Study on antitumor effect of koumine. Pharmacol Clin Chin Mater Med. 2006;22(5):6-8.

39. Wang $Y$, Fang $Y$, Lin W, Cheng $M$, Jiang $Y$, Yin M. Inhibitory effect of gelsemium alkaloids extract on hepatic carcinoma HepG2 cells in vitro. J Chin Med Mate. 2001;24(8):579-81.

40. Huang J, Xu Y, Yu C, Xie R. Antitumor effects and mechanisms of alkaloidal compounds from Gelsemium Elegans Benth. J Strait Phar. 2014;26(10):19-23.

41. Zhang R, Zhu X, Bai H, Ning K. Network pharmacology databases for traditional Chinese medicine: review and assessment. Front Pharmacol. 2019;10:123. https://doi.org/10.3389/fphar.2019.00123.

42. Duan B, Han L, Ming S, Li J, Wang Q, Zhang D, et al. Fuling-Guizhi herb pair in coronary heart disease: integrating network pharmacology and in vivo pharmacological evaluation. Evid Based Complement Alternat Med. 2020; 2020:1489036.

43. Cao HY, Xiao CH, Lu HJ, Yu HZ, Hong H, Guo CY, et al. MiR-129 reduces CDDP resistance in gastric cancer cells by inhibiting MAPK3. Eur Rev Med Pharmacol Sci. 2019;23(15):6478-85. https://doi.org/10.26355/eurrev_201 908_18531.

44. Du Y, Zhang J, Meng Y, Huang M, Yan W, Wu Z. MicroRNA-143 targets MAPK3 to regulate the proliferation and bone metastasis of human breast cancer cells. AMB Express. 2020;10(1):134. https://doi.org/10.1186/s13568-02 0-01072-w.

45. Yu T, Wang CY, Tong R. ERBB2 gene expression silencing involved in ovarian cancer cell migration and invasion through mediating MAPK1/ MAPK3 signaling pathway. Eur Rev Med Pharmacol Sci. 2020;24(10):5267-80. https://doi.org/10.26355/eurrev_202005_21309.

46. Baba Y, Nosho K, Shima K, Meyerhardt JA, Chan AT, Engelman JA, et al. Prognostic significance of AMP-activated protein kinase expression and modifying effect of MAPK3/1 in colorectal cancer. Br J Cancer. 2010;103(7): 1025-33. https://doi.org/10.1038/sj.bjc.6605846.

47. Qi L, Ding Y. Construction of key signal regulatory network in metastatic colorectal cancer. Oncotarget. 2018;9(5):6086-94. https://doi.org/10.18632/ oncotarget.23710.

48. Vaseghi Maghvan P, Rezaei-Tavirani M, Zali H, Nikzamir A, Abdi S, Khodadoostan $\mathrm{M}$, et al. Network analysis of common genes related to esophageal, gastric, and colon cancers. Gastroenterol Hepatol Bed Bench. 2017; 10(4):295-302.

49. Chen D, Song S, Lu J, Luo Y, Yang Z, Huang Q, et al. Functional variants of $-1318 \mathrm{~T}>\mathrm{G}$ and $-673 \mathrm{C}>\mathrm{T}$ in c-Jun promoter region associated with increased colorectal cancer risk by elevating promoter activity. Carcinogenesis. 2011;32(7):1043-9. https://doi.org/10.1093/carcin/bgr047.

50. Wang SY, Gao K, Deng DL, Cai JJ, Xiao ZY, He LQ, et al. TLE4 promotes colorectal cancer progression through activation of JNK/c-Jun signaling pathway. Oncotarget. 2016;7(3):2878-88. https://doi.org/10.18632/oncota rget.6694.

51. Ren Z, Yao L, Liu J, Qi Z, Li J. Silencing NOB1 can affect cell proliferation and apoptosis via the C-Jun N-terminal kinase pathway in colorectal cancer. J Invest Surg. 2020. p. 1-7. https://doi.org/10.1080/08941939.2019.1697401.
52. Liu Y, Chen X, Cheng R, Yang F, Yu M, Wang C, et al. The Jun/miR-22/HuR regulatory axis contributes to tumourigenesis in colorectal cancer. Mol Cancer. 2018;17(1):11. https://doi.org/10.1186/s12943-017-0751-3.

53. Saif MW. Colorectal cancer in review: the role of the EGFR pathway. Expert Opin Investig Drugs. 2010;19(3):357-69. https://doi.org/10.1517/13543781 003593962.

54. Li J, Wang Y, Wang X, Yang Q. CDK1 and CDC20 overexpression in patients with colorectal cancer are associated with poor prognosis: evidence from integrated bioinformatics analysis. World J Surg Oncol. 2020;18(1):50. https:// doi.org/10.1186/s12957-020-01817-8.

55. Gan W, Zhao H, Li T, Liu K, Huang J. CDK1 interacts with iASPP to regulate colorectal cancer cell proliferation through p53 pathway. Oncotarget. 2017; 8(42):71618-29. https://doi.org/10.18632/oncotarget.17794.

56. Yuan Z, Liang Z, Yi J, Chen X, Li R, Wu J, et al. Koumine promotes ROS production to suppress hepatocellular carcinoma cell proliferation via NFkappaB and ERK/p38 MAPK signaling. Biomolecules. 2019;9(10):559. https:// doi.org/10.3390/biom9100559.

57. Ardito F, Giuliani M, Perrone D, Troiano G, Lo ML. The crucial role of protein phosphorylation in cell signaling and its use as targeted therapy (review). Int J Mol Med. 2017;40(2):271-80. https://doi.org/10.3892/ijmm.2017.3036.

58. Fang JY, Richardson BC. The MAPK signalling pathways and colorectal cancer. Lancet Oncol. 2005;6(5):322-7. https://doi.org/10.1016/S1470-204 5(05)70168-6.

59. Luo H, Hao E, Tan D, Wei W, Xie J, Feng X, et al. Apoptosis effect of Aegiceras corniculatum on human colorectal cancer via activation of FoxO signaling pathway. Food Chem Toxicol. 2019;134:1 10861. https://doi.org/1 0.1016/j.fct.2019.110861.

60. Danielsen SA, Eide PW, Nesbakken A, Guren T, Leithe E, Lothe RA. Portrait of the PI3K AKT pathway in colorectal cancer. Biochim Biophys Acta. 2015; 1855(1):104-21. https://doi.org/10.1016/j.bbcan.2014.09.008.

61. Dong YX, Pang ZG, Zhang JC, Hu JQ, Wang LY. Long non-coding RNA GClnc1 promotes progression of colorectal cancer by inhibiting p53 signaling pathway. Eur Rev Med Pharmacol Sci. 2019;23(13):5705-13. https:// doi.org/10.26355/eurrev_201907_18308.

62. Slattery ML, Mullany LE, Wolff RK, Sakoda LC, Samowitz WS, Herrick JS. The p53-signaling pathway and colorectal cancer: interactions between downstream p53 target genes and miRNAs. Genomics. 2019;111(4):762-71. https://doi.org/10.1016/j.ygeno.2018.05.006.

63. Chen B, Xia Z, Deng YN, Yang Y, Zhang P, Zhu H, et al. Emerging microRNA biomarkers for colorectal cancer diagnosis and prognosis. Open Biol. 2019; 9(1):180212. https://doi.org/10.1098/rsob.180212.

64. De Simone V, Franze E, Ronchetti G, Colantoni A, Fantini MC, Di Fusco D, et al. Th17-type cytokines, IL-6 and TNF-alpha synergistically activate STAT3 and NF-kB to promote colorectal cancer cell growth. Oncogene. 2015; 34(27):3493-503. https://doi.org/10.1038/onc.2014.286.

65. Yuan Z, Matias FB, Wu J, Liang Z, Sun Z. Koumine attenuates lipopolysaccaride-stimulated inflammation in RAW264.7 macrophages, coincidentally associated with inhibition of NF-kappaB, ERK and p38 pathways. Int J Mol Sci. 2016;17(3):430.

66. Su YP, Shen J, Xu Y, Zheng M, Yu CX. Preparative separation of alkaloids from Gelsemium elegans Benth. Using pH-zone-refining counter-current chromatography. J Chromatogr A. 2011;1218(23):3695-8. https://doi.org/10.1 016/j.chroma.2011.04.025.

67. Chi D, Lei L, Jin H, Pang J, Jiang Y. Study of koumine-induced apoptosis of human colon adenocarcinoma Lovo cells in vitro. J First Mil Med Univ. 2003; 23(9):911-3.

68. Xu Y, Qiu HQ, Liu H, Liu M, Huang ZY, Yang J, et al. Effects of koumine, an alkaloid of Gelsemium elegans Benth., on inflammatory and neuropathic pain models and possible mechanism with allopregnanolone. Pharmacol Biochem Behav. 2012;101(3):504-14. https://doi.org/10.1016/j.pbb.2012.02. 009.

\section{Publisher's Note}

Springer Nature remains neutral with regard to jurisdictional claims in published maps and institutional affiliations. 